\title{
Intuition, insight, and the right hemisphere: Emergence of higher sociocognitive functions
}

This article was published in the following Dove Press journal:

Psychology Research and Behavior Management

26 February 2010

Number of times this article has been viewed

\author{
Simon M McCrea \\ Departments of Neurology and \\ Neuroophthalmology, University \\ of British Columbia, Vancouver, \\ British Columbia, Canada
}

Correspondence: Simon M McCrea Departments of Neurology and Neuroophthalmology, University of British Columbia, Vancouver, British Columbia, Canada Emailsmccrea@interchange.uba.ca
Abstract: Intuition is the ability to understand immediately without conscious reasoning and is sometimes explained as a 'gut feeling' about the rightness or wrongness of a person, place, situation, temporal episode or object. In contrast, insight is the capacity to gain accurate and a deep understanding of a problem and it is often associated with movement beyond existing paradigms. Examples include Darwin, Einstein and Freud's theories of natural selection, relativity, or the unconscious; respectively. Many cultures name these concepts and acknowledge their value, and insight is recognized as particularly characteristic of eminent achievements in the arts, sciences and politics. Considerable data suggests that these two concepts are more related than distinct, and that a more distributed intuitive network may feed into a predominately right hemispheric insight-based functional neuronal architecture. The preparation and incubation stages of insight may rely on the incorporation of domain-specific automatized expertise schema associated with intuition. In this manuscript the neural networks associated with intuition and insight are reviewed. Case studies of anomalous subjects with ability-achievement discrepancies are summarized. This theoretical review proposes the prospect that atypical localization of cognitive modules may enhance intuitive and insightful functions and thereby explain individual achievement beyond that expected by conventionally measured intelligence tests. A model and theory of intuition and insight's neuroanatomical basis is proposed which could be used as a starting point for future research and better understanding of the nature of these two distinctly human and highly complex poorly understood abilities.

Keywords: intuition, insight, nonverbal decoding, nonverbal sequencing, unconscious and conscious processes, right hemisphere dominance, atypical localization of cognitive functions, crossed aphasia, inverse cognitive modeling, emergent properties, anomalous functions, specialization, visual gesture lexicon, crosslinguistic fluency, achievement-ability discrepancy, IQ threshold theory, functional capacity, House-Tree-Person, drawings, clinical intuition, clinical psychology

“It's not that I'm so smart, it's just that I stay with problems longer"

—Albert Einstein

"The right hemisphere lacks the capacity to generate productive language in over 95\% of the population... [therefore most research]...focused on the language capabilities of this 'typical' right hemisphere... (p. 132)."' This comment centred on studies of the 'typical' right hemisphere in the majority of subjects' for whom the left hemisphere is language dominant. Right hemisphere language functions were revealed by: commissurotomy, epilepsy lesion resection, hemispherectomy, stroke or in cases of brain injury. This review also listed right hemisphere-dependent processes which parallel 
left hemisphere-dependent language processes. To name a few would include: visual word recognition, pre-lexical influences on encoding, phonology, orthography, imageability, syntax and semantics, deep dyslexia and letter-by-letter reading. ${ }^{2}$ However as with most research before it on this topic all patients were previously severely neurologically compromised. Hence it would be erroneous and inappropriate to use these defective populations for the study of advanced putative right hemisphere cognitive processing modules or 'traits'. This would be all the more true for those right hemisphere cognitive functions operating at the upper level of complexity responsible for instantiating neurally complex human social interaction and communication. This fact is due to the ostensibly deleterious effects of any neurological lesion on higher cortical functions. ${ }^{3}$ The contrary and still widely held neurolinguistic doctrine on right hemisphere language functions is that the dominant left hemisphere alone demonstrates an intrinsic superiority at linguistic mediation. ${ }^{4}$ However again many of these old neurolinguistic assumptions consisted of arguments, studies, and theory that were based solely on inherently pathological cases and without the benefit of modern functional neuroimaging technologies.

To advance a more specific hypothesis: Are endogenous differences to be found in atypical right hemisphere localized subjects where strong hemispheric dominance exists that is not complicated by mixed dominance or pre-existing pathology? Strongly atypically localized and unilateral right hemisphere language dominant subjects are the main focus of this article (eg, see Floel and colleagues). ${ }^{5}$ The hypothesis here is that the co-association of language along with other novel module combinations to the nondominant hemisphere may precipitate development of emergent higher-order cognitive functions. The hypothesis is based on a dozen case reports of strongly right-hemisphere lateralized subjects with average general ability scores and yet with superior indices of achievement. ${ }^{5-8}$ A large achievement and aptitude test difference implies that some other ability may be mediating the usually strong correlation of achievement and ability test concordance. Modern functional neuroimaging studies are becoming available which are not confounded by the problem of pre-existing lesions or neuropathology.

Some new structural neuroimaging studies such as diffusion tensor imaging (DTI) are being carried out that allow researchers to examine why a specific neural tract might be expected to divert along a specific neural trajectory ontogenetically and developmentally. These studies may go far in explaining how a specific neural functional architecture could support exceptional instances of intuitive acumen and/or insight in problem solving. For instance, recent DTI studies show that a "ventral semantic stream" consisting of the fast conducting inferior occipitofrontal fasciculus fibers links the occipitotemporal and orbital regions within the dominant hemisphere. This newly discovered tract may play a key role in multiple concept semantic mapping ${ }^{9}$ that has been posited in extensive reviews of the right hemisphere's parsing of complex discourse functions. ${ }^{10}$ Incidentally, as we will see this neural tract also possesses spatiotemporal properties that could function as a conduit linking intuition and insight. Complex discourse functions might include for instance mapping the common threads of discussion between articles appearing in a newspaper over the course of a week of publications. Such architectures might be able to synchronize widespread schemas within the cortex and provide a type of "semantic gestalt" along the lines of how perceptual gestalts are put together in the posterior sensory processing cortices. ${ }^{11}$

The theory of co-localization and emergent cognitive functions was previously hypothesized by Basso and colleagues. ${ }^{12}$ Co-localization suggests that unique combinations and permutations of modules may reside in one hemisphere or another or even perhaps in the anterior or posterior components within a hemisphere. Emergent properties suggest cognitive processes that are qualitatively different from each other, either in terms of structure and/or function. Complete reversibility in the lateralization of cognitive functions has been previously reported. It is however, quite rare $^{13}$ and has yet to be studied systematically with functional and structural neuroimaging or even using in depth neuropsychological assessment and longitudinal achievement studies. There are almost no published studies of atypical right hemisphere language dominants subjects where comprehensive neuropsychological assessments were carried out in conjunction with in depth structural and functional neuroimaging studies.

Given the inherent level of complexity in cognitive modules that are based within an entire hemisphere (or that are the product of whole brain volume oscillation of synchronized signals), comprehensive neuropsychological single case studies and field studies of the social aspects of these phenomenon would be most appropriate. ${ }^{14}$ Comprehensive cataloguing of single-case studies of such phenomenon in atypical subjects are nonexistent. Participant-observation studies of such putative advanced social signaling communicative abilities in atypically localized subjects cannot be found in the published literature. In addition to the academic and practical implications 
of atypical localization for cognitive neuropsychology there are profound theoretical implications for neurolinguistics and theories of language ontogeny in humans. Atypical localization cases or unique co-localization scenarios can be instructive in understanding the fundamental and deep principles by which the cerebral cortex is organized, with respect to language in association with other major neurocognitive functions. ${ }^{15}$ Basso and colleagues was among the first to note the fruitfulness of such questioning. ${ }^{12}$

Hence, given the above, no theory about the organization of language or cognitive function can be considered comprehensive and/or "complete" without inclusion of a theory that accounts for the "anomalies". Indeed the single case in neuropsychology is enough by itself to tell experimenters and researchers what the human brain is capable of (or not capable of for that matter!) as well as providing for the basis for generalizations to the population as a whole. ${ }^{16} \mathrm{~A}$ full accounting of how these atypical and anomalous cases of right hemisphere language arise should illuminate the genetic, evolutionary, and functional aspects of these complex, rare and emergent properties. A complete accounting of such anomalies in any comprehensive cognitive neuroscience of language should help to establish the upper limit parameters on what constitutes language by nature as well as the natural constraints placed on the development of such linguistic processes. Such studies could be instructive on how normal language evolved and for what purposes language evolved in the first place in ancestral human beings.

From a more mechanistic point of view, Fischer and colleagues specifically enquired how different cognitive and behavioral operations might be related to each other in terms of functional outcome. ${ }^{13}$ That is, is it possible that the intrinsic characteristics or cognitive functions of unexpectedly displaced modules transforms as a function of either (i) dislocation, or (ii) co-localization, along with other modules, within an unexpected hemisphere? Is it true that atypicality and anomalous language representation and unique co-localization of other cognitive functions can result in a functional configuration of neural networks that is favorable to the exceptional development of higher-order cognitive functions such as intuition and insight? Can completely unique cognitive or sociocognitive abilities arise developmentally in such anomalous individuals? How would any such rare and new abilities that are sparingly distributed (or patently not even present), in the general population relate structurally and functionally to other abilities such general intelligence or spatial abilities? It is hypothesized that right-ward language localization and unique co-localization configurations are unique and that such cases can illustrate fundamental principles of language organization even in the normal brain.

\section{Organization of this review}

In this extensive theoretical overview of the putative higherorder functions instantiated in part by the right hemisphere a diverse selection of cognitive psychological, neurological, neuroradiological, and neuropsychological literature and data will be examined. Initially we will undertake to define intuition and insight in practical, historical and experimental terms. Secondly we will show how intuition and insight are intrinsically nested within social-cultural constructs of society and to notions of collective mind. Thirdly we will show how intuition and insight constructs are related to each other through empirical studies in the cognitive sciences. Fourthly, we will show how nonverbal decoding efficacy appears to be a central construct in understanding intuition as a function of cognitive psychological expertise.

As we will see conscious and unconscious processes and their integration with emotional evaluation will be central to understanding the link between intuition and insight. Focusing almost entirely on contemporary functional neuroimaging studies (eg, functional magnetic resonance imaging [fMRI], position emission tomography [PET] and magnetoencephalography [MEG]) we will discuss the neural correlates of intuition first which may have a more bilateral representation. Then we will discuss the largely right hemispheric networks underlying insight using many of the same techniques. This two-component network likely figures prominently in virtually all complex novel social problem-solving and thus would likely confer selective adaptive survival value. We will then endeavor to briefly summarize how the self may be a central concept mediating between unconscious and conscious mind; and hence between intuition and insight. Carl Jung conceived of the self as representing the quest for unity and integration in personality and he held that the self as a concept was not yet mature until the third decade of life. ${ }^{18}$ In Jung's 1928 theory the "self" was a midpoint between the conscious and unconscious minds. ${ }^{19}$

With this conceptual overview of how conscious and unconscious processes are interrelated we can then focus on the interesting case of how reversed linguistic asymmetry can shed light on many theoretical neurolinguistic assumptions and hypothesized concepts. There is indeed good evidence that a right ventral hemispheric network operates in both intuition and insight mediated in part perhaps by lateralized caudate nucleus and orbitofrontal networks. In the third part of the review we undertake to show how crossed aphasia 
was the initial impetus for all early conceptualizations of the functions of the right hemisphere. For many contemporary reasons it can be shown that this standard view of the neurolinguistic inferiority of the right hemisphere is now doubtful and should be viewed with scepticism. Praxis lateralization (either in oppositional hemispheres or co-localized within the right hemisphere with language in atypicals) is discussed as an effect perhaps mediated by the posited "right shift" gene. Such a gene might have enabled language production and comprehension for group communication simultaneous with superior unimanual praxis in ancestral conditions.

A hypothetical line of argumentation shows that atypicality may confer adaptive traits and those anomalous patterns of resting state functional lateralization of intuition and insight could be readily explained. A set of subjects with strong rightward language lateralization that demonstrate characteristic intelligence quotient (IQ)achievement discrepancies are suggested to be examples of individuals with enhanced or heightened intuitive and insightful processing functions. Although this set of atypical subjects is a small sample that has not been extensively evaluated, in the context of the other lines of converging evidence the summary results are provocative. The review ends with a practical discussion for future research studies on this neglected topic. In the first instance extreme individual differences in these sociocognitive functions may necessarily exist with some rather interesting implications. In the second instance there may not only be quantitative differences in performance of such real-world intuitive and insightful sociocognitive functions - but due also to emergent properties - there may be qualitative differences in the functional neural architecture associated with anomalous or atypical subjects. It is also shown how these sociocognitive functions may be currently poorly understood because of the necessity of studying them in interaction within the larger sociocultural milieu as in participant-observation or field studies. This is a highly complex quasi-experimental approach fraught with inherent measurement difficulties as well as concept operationalization problems. There are also more cognitive neuropsychological implications for understanding gender differences, the nature of intelligence, and the proper psychometric assessment of these putative higher-order abilities as well as understanding the functioning of mixed cortical and subcortically-based neural networks. Finally a clinically relevant and practical example of intuitive-insight based acumen from clinical psychology is put forward with an associated specific set of testable assumptions and hypotheses for future research in this area.

\section{Definition of intuition and insight}

According to the Oxford English Dictionary, insight is “... the capacity to gain an accurate and deep intuitive understanding of something..." ${ }^{20}$ Gestalt psychologist and theoretical linguist, Karl Bühler, originally coined the term insight and here it is conceived of as an individual differences variable that people may possess in varying quantities. ${ }^{21}$ In contrast to intuition, insight involves a period of incubation of the problem before the recognition of a solution. ${ }^{22}$ There is usually the emergence of a specific temporal pattern associated with the solution to the problem as the solution becomes more and more conscious ${ }^{23}$ culminating in the 'aha or eureka moment'. ${ }^{24}$ Intuition, in contrast, has been described by Knoblich as "...the ability to understand something immediately, without the need for conscious reasoning..." There are key differences between intuition and insight. Intuition occurs instantly and is emotionally laden; it does not have the accompanying verbal, conscious declarative awareness of the final stage of insight. ${ }^{25}$ Interestingly, from the point of view of the cognitive and neural systems supporting the two higher-order processes, is the finding that intuition often precedes an actual conscious insight. ${ }^{26}$ This stage-like aspect of intuition and insight as conceived of in this theoretical review will be elaborated on in subsequent sections. Bechara and Damasio similarly described intuition as a holistic hunch, "a gut feeling" or a sense of absolute certainty or awareness that a perception is on the edge of awareness. ${ }^{27}$ Insight and intuition are variables that should have a normal distribution within the general population.

Finally, a social cognitive neuroscience perspective of intuition has been found incompatible with the self-report of intuitive "cognitive style" exemplified by the Myers-Briggs type indicator (MBTI). ${ }^{28}$ In their review of empirical studies focused on the concept of intuition, Hodgkinson and colleagues $^{29}$ noted that none of the sensing-intuition scale items of the MTBI assessed affective or behavioral aspects of the concept, as recently defined by social cognitive neuroscientists. ${ }^{30}$ Moreover, Hodgkinson and Clarke found that the constructs on which the MTBI are based are theoretically incompatible with Carl Jung's theory and suggest behavioral predictions which are contrary to central tenets of the theory. ${ }^{31}$ This raises questions about its suitability for the assessment of intuition psychometrically and in particularly the construct validity of the MTBI as a whole. Nonetheless in this review Jung's early writings ${ }^{19}$ on the 
structure of the self per se do play a considerable role in our agentic construct of intuition and insight. Similarly, a major difference then between insight and intuition is the degree to which the two related constructs are declarative/ explicit or nondeclarative/implicit. Intuition is by definition nondeclarative and implicit, whereas insight can be declarative and explicit in memory.

Lieberman's review on intuition makes important distinctions between the two terms. ${ }^{30}$ Thus, intuition differs from the 'eureka moments' characteristic of insight:

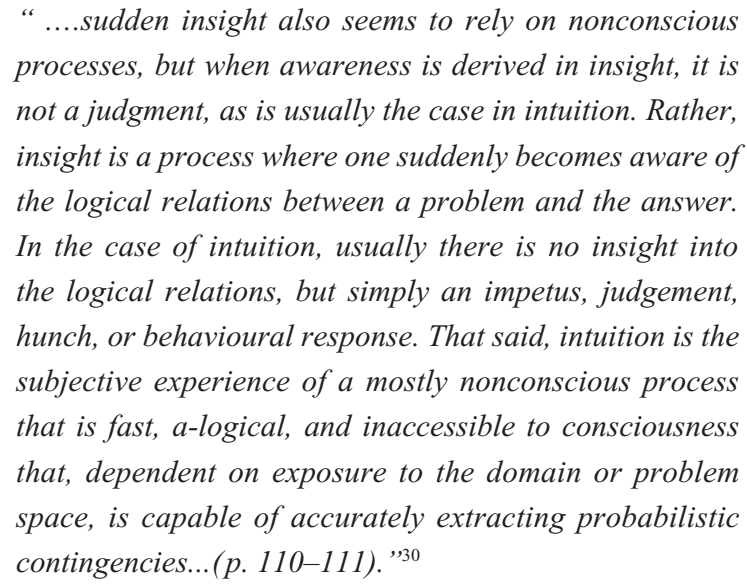

Bruner $^{32}$ noted that intuition"...rests on familiarity with the domain of knowledge and with its structure..." (p. 57) $)^{32}$ which is a definition congruent with cognitive psychological expertise. Recent work has shown that the ability to intuit is acquired through experience and learning within a specific domain, and indeed appears to be particularly associated with perceptual pattern recognition processes. ${ }^{29}$ Highly skilled intuiters have been found capable of holding many sets of patterns pertaining to a domain of knowledge in their immediate memory, along with information about the emotional salience of each piece of information. ${ }^{33}$ Hence, the relationship between intuitive behavior and expert experience within a domain of knowledge, is especially germane. For instance, a chess master may hold 50,000 chess patterns in long-term memory. ${ }^{33}$ Similarly, as an example, it is hypothesized that veteran homicide detectives may have an intrinsic feel for the truthfulness of a suspect's story based on the experience of conducting hundreds of previous interviews, and utilizing a collection of techniques. Finally, Ericsson and Charness noted that, consistent with the intuition and expertise within a domain hypothesis, systematic studies reveal that it requires 10 or more years of experience and dedicated practice with skilled apprenticeship, to become a true expert within a specific domain of knowledge. ${ }^{34}$ In this sense intuition and expertise both carry with them complex pattern recognition processes, with an intrinsic motor component, the routinization of which suggests a subcortical basal ganglia loci of action.

\section{Social dimensions of intuition and insight}

Since intuition and insight both carry with them connotations of significant interaction with the sociocultural milieu a brief review of current research in this area is advisable. Intuition and insight are usually defined in terms of their salience for scientific discovery. Unfortunately this perspective is often associated with a singular person view of history. This view of the origins of intuition and insight fails to encompass the importance of specific cognitive functions in activities of daily living; especially of a social nature. In other words, from a practical and utilitarian point of view, intuition and insight may play an essential role in the pragmatic functions of language and communication. By pragmatics we mean "... the way that language is used and interpreted while taking into consideration the characteristics of the speaker and hearer and the effects of contextual and situational variables... (p. 175)."15 For the detective the way a suspect responds to a line of questioning through gesture or prosody can be interpreted to mean that they are telling the truth or are lying. As we will soon discuss it is such nonverbal decoding of human behavior in face-to-face interactions and perhaps at some distance that may play a central role in any conceptualization of what these abilities are in essence. Interestingly, neuropragmatics or the study of how the brain comprehends and produces linguistic behavior, shares many similarities with the underlying functional neuroanatomical systems associated with intuition and insight. ${ }^{35}$ Hierarchical discourse processes and unstated assumptions; indirect requests; figurative, metaphoric and model building language; mentalizing and theory of mind; and neurolinguistic right hemisphere functions are all examples of a neuropragmatic and intuition-insight link. ${ }^{10}$

This functional, socially relevant, and practical view of insight and intuition is the one that we propose to utilize for model-building and hypotheses generation in social cognitive neuroscience. ${ }^{29}$ In their reviews Hodgkinson and colleagues as well as Lieberman note that intuition and insight offer considerable explanatory power as essential bridging constructs in social cognitive neuroscience. ${ }^{29,30}$ Intuition and insight seem to be able to bridge the individual psychology of the thinking variables (eg, cognitive science) with the more contextualized nature of social psychology. In this view, the fundamentally social elements of intuition and insight are inseparable from concise definitions of the two terms and their theoretical interrelations. As such they are more truly 
conceptualized as social rather than cognitive psychological processes. Csikszentmihalyi and Sawyer note that many cultures have words for such concepts as insight, which are by nature exceptional and highly valued within a culture. ${ }^{36}$ Moreover, these authors note that basic stages are associated with insight. In the first stage, an insight is associated with a state of mind relevant to a particular topic, or to knowledge which may be known as a developed domain of expertise. Secondly, a moment of realization results as a consequence of the acquisition of new information specific to the knowledge domain. Finally, a quick re-structuring of the model (as in the example of a "semantic gestalt" where the picture fits with many lines of different evidence), is subjectively experienced as new understanding.

When Csikszentmihalyi and Sawyer interviewed objectively determined exceptionally creative women and men (eg, Nobel Prize winners), a characteristic four stage narrative model of interactional factors emerged. ${ }^{36}$ The first stage consisted of laborious reading and research preceding the insight. The second stage was a period of idle time usually spent alone, with the critical third stage of the insight occurring almost momentarily. Finally, the fourth stage consisted of the work-intensive fleshing out of ideas, and painstaking elaboration in order to bring the idea to fruition. Hence for these authors the 'hard work' that actually precedes insight in scientific and artistic endeavors is usually deeply rooted in interaction with colleagues, and the individual's internalized understanding of a culturally constituted domain. However from a more practical point of view, it is generally accepted that some individuals develop more expertise in social understanding and socially productive behavior than others. That is, do some people differ, in a manner similar to an individual difference variable, in the amount of raw intuition and insight that they possess in understanding other people? If so, by what emotional, cognitive, and neuropsychological mechanisms could fundamentally socially based raw intuition and insight develop?

In their interviews, Csikszentmihalyi and Sawyer found that creative individuals displayed strong subjective awareness of external social or disciplinary influences with reference to (i) interpersonal contact (ii) strategic considerations, and (iii) awareness of the relevant paradigms. ${ }^{36}$ Furthermore they possessed an awareness of the central questions occupying the consciousness of the field, a keen appreciation for the zeitgeist or "spirit of the times". An additional creative process set of variables uncovered through the interviews was an iterative loop recurring from the individual person (constituting evolutionary pattern of variation) to selection (the field in which the person works) to retention (or the cumulative record), consisting of a communicable symbolic product. Similarly, two levels of insight were discovered. The first mini-insights might occur in the afternoon following relevant reading in the morning. The second paradigmchanging insight was characteristically much deeper and more profound, often with untold implications across many spheres of knowledge. The latter type of insight is exemplified by Darwin's many years spent analyzing hundreds of previously undescribed species which thereafter culminated in his formulation of the theory of natural selection. The evaluative and elaborative process associated with this deep type of insight involves integration with the discipline and the larger domain of knowledge. In extreme creativity an unintended result maybe the complete overthrow of existing tacit assumptions within and across disciplines. ${ }^{37}$

Deep long-term insight and shorter-term mini-insight can further be distinguished on the basis of the type of problemsolving required. ${ }^{38} \mathrm{~A}$ presented problem-solving process occurs over a short-time frame where a problem is known and pre-existing in the domain. In contrast, the discovered problem-finding process is much longer term, typically occurring over years of study and often with ill-defined parameters and types of problems. In cases of discovered problem-finding processes the actual problem may not be recognized (become conscious), until the moment of insight. Incidentally, in this review, as we will discuss, ill-defined problems (and also associated with discovered problem-finding insightful processes), are highly sensitive to right hemisphere prefrontal cortex lesions. ${ }^{39}$ Paradigmatic shifts belong to this latter category of insight. When we speak of insight in the layperson's understanding of the term, it appears that intuition is related to insight specifically through this incubation stage (Figure 1). That is, the incubation stage in discovered problems seem to be based on the random convergence and amalgamation of ideas (or in our terminology "intuitive schemas") from different disciplines, sometimes facilitated, but not always, by interaction with individuals from those fields. ${ }^{36}$

Hence, due to the sheer difference in time scales and orders of complexity between presented and discovered problems, problem finding creativity will make greater use of subconscious processing. ${ }^{36}$ Therefore, the preparation stage will have a crucial social dimension encompassing apprenticeship, mentoring, solitary study and interaction with experts, which is conscious, declarative, social and serial in nature. In contrast, the incubation stage will allow for multiple chunks of dense information units or models to be integrated simultaneously in a generate, test, and predict sequence. 


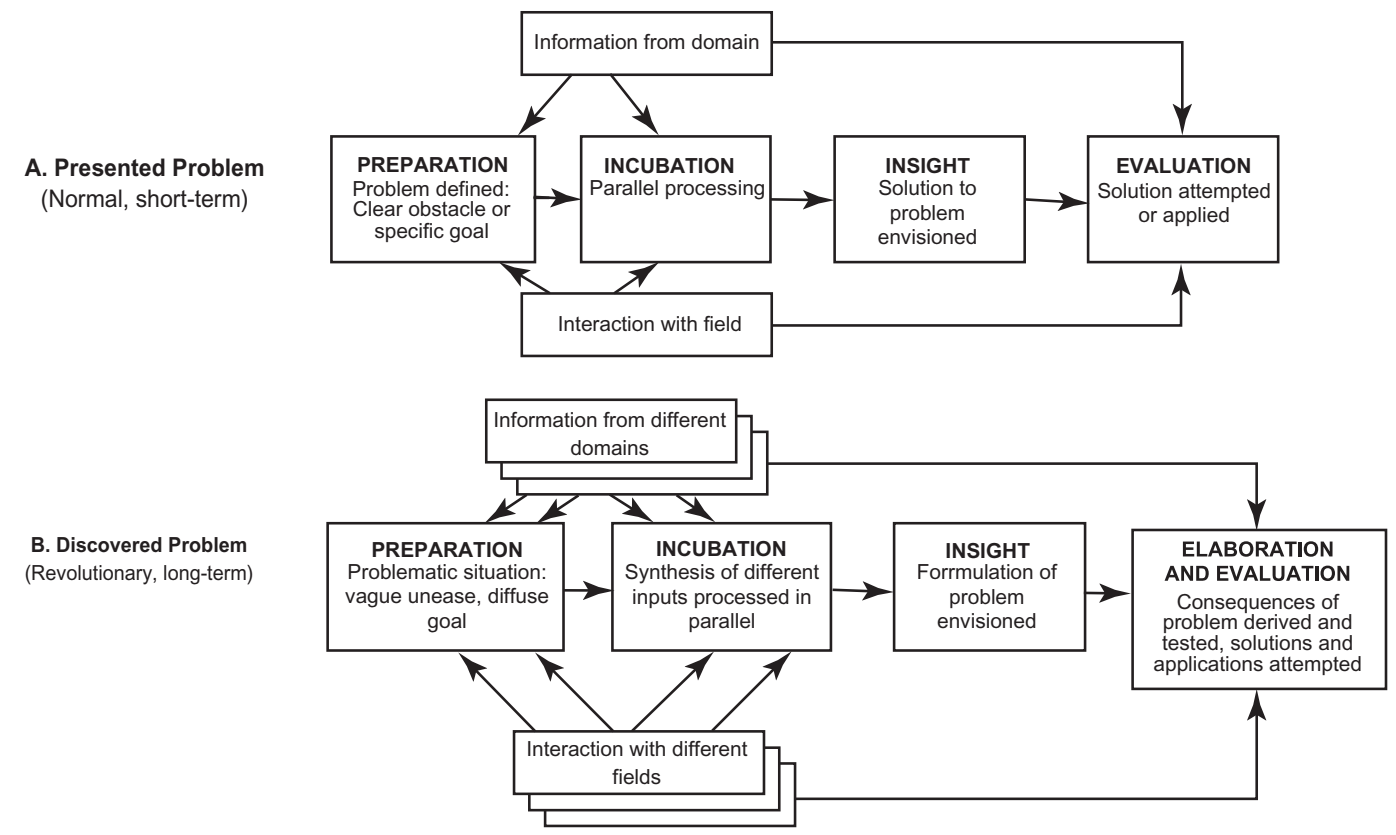

Figure I A long-term and slow route to creative insight. Copyright $\odot$ 1995, The MIT Press. Adapted from Csikszentmihalyi M, Sawyer K. Creative insight: The social dimension of a solitary moment. In: Sternberg RJ, Davidson JE, editors. The Nature of Insight. Cambridge, MA: The MIT Press; 1995. p. 329-363.

In Figure 1, we can see where in the model intuition will fit. As to be examined in subsequent neuropsychology sections, intuition, with its subconscious and automatic features would be likely associated with subcortical and limbic connectivity. Intuition's automatic and highly routinized nature would fit within the high processing bandwidth incubation stage, where complex domain-specific models can be generated, tested and evaluated. In this context, deep knowledge and heuristic structures could be fitted and tweaked until a new paradigm emerges into consciousness. The final stage would consist of laborious step-wise experimentation, characteristic of normal Kuhnian science, that brings a concept, idea, or hypothesis to fruition.

In interviews with 100 eminent artists, writers, scientists, and public institution leaders seven prerequisites for longer-term problem-finding syntheses were noted. ${ }^{36}$ The creative, insightful individual must have: “...(i) a thorough knowledge of one or more symbolic domains; (ii) thorough immersion in a field that practices the domain; (iii) focus of attention on a problematic area of the domain; (iv) ability to internalize information relevant to the problematic area; (v) ability to let the relevant information interact with information from other domains at a subconscious level where parallel processing takes place; (vi) ability to recognize a new configuration emerging from this interaction that helps resolve the problematic situation; and (vii) evaluation and elaboration of the insight in ways that are understandable and valuable to the field... (p. 358-359).."36 Hence, we can observe that there are already plausible theoretical links between objectively defined intuition and insight, and an examination of some of the empirical data will further clarify our understanding.

\section{Cognitive science, intuition and insight}

The eureka or aha moment of insight was aptly described as the illumination stage, ${ }^{40}$ and some of the earliest theories of insight directly noted that an insight was often preceded by an intuition. Metcalfe and Wiebe found that noninsight problems (eg, algebra, analytical thinking, logical thinking) were open to accurate self-predictions of performance by subjects, whereas insight problems were not. ${ }^{41}$ Similarly, whereas analytical problems were solved incrementally over time researchers confirmed that insight problems were solved with "an opaque leap". Wallas directly likened this "illumination stage" to a train or sequence of associations which alludes to the role of motor sequencing in the incubation of intuitions associated with subsequent insight. Incidentally, sequencing has been associated with the integrity of the frontal-temporal lobe ${ }^{42}$ and the associated subcortical basal ganglia structures. This sequencing and ostensibly motor nature of incubation of intuitions and their transformations into insight will be elaborated further in subsequent sections of this review. 
Dorfman and colleagues suggested that intuition and incubation reflect unconscious processing, which are both aspects of implicit thought. ${ }^{43}$ The concept of implicit learning is essential to understanding the illumination stage; and it is defined as any change in experience, thought, or action sequence that is attributable to the acquisition of new semantic or procedural knowledge. This acquisition of knowledge occurs under the conditions of the absence of conscious awareness of that knowledge. In implicit cognition studies there are two related important constructs: the objective and subjective threshold. The objective threshold is the point at which differential motor response to a stimulus disappears, considered as an objectively defined Skinnerian "emitted" motor threshold. In contrast the subjective threshold is the point at which the stimulus cannot be consciously perceived, usually as indicated by verbal report. Deep semantic processing is possible for stimuli presented at subjective threshold, whereas the motor response associated with objective threshold usually is associated with shallow perceptual processing. ${ }^{44}$

Experimental evidence that intuition is directly related to insight is chronicled in many studies. ${ }^{45}$ The remote associates test is the classic example of insight problem solving ${ }^{46}$ however it has also recently been used with much success to study intuition. ${ }^{47,48}$ In the remote associates test or RAT word triads are presented to subjects such that the three words are semantically related to each other in terms of a common fourth target word. Typically, this fourth target word is activated automatically through spreading activation of semantic networks which converges through summation priming on the remote associate. ${ }^{49,50}$ Topolinski and Strack have adapted this strategy of the RAT for the objective study of intuition by requiring subjects to simply tell whether they can predict whether the RAT item is soluble or not. ${ }^{47}$ This engenders that subjects provide a response before 2000 milliseconds and subjects can actually recollect a feeling of knowing that they either can solve, or not solve, the triad even when they do not know the answer to the triad. Subjects can perform this intuitive version of the RAT task in less than $2000 \mathrm{msec}$ significantly above chance and thus Topolinski and colleagues have succeeded in directly linking intuition and insight in a superb experimental model.

In the first instance, when two sets of remote association items were provided to subjects, purposely only one triad could be solved. ${ }^{45}$ In the absence of a solution, a judgment of which triad was coherent or soluble represented only a hunch or intuition. Nonetheless, subjects were able to choose which triads were soluble at rates greater than chance.
In the second experiment, sets of remote associate test triads were either semantically convergent (eg, goat, pass, green: solution $=$ mountain $)$ or semantically divergent (eg, strike, same, tennis: solution $=$ match). Congruent with a subconscious semantic priming effect, the semantically convergent triad was more likely to be guessed as coherent or soluble. ${ }^{45}$ A third experiment illustrated the potential relationship between pictorial and verbal perceptual matching in the intuition-insight connection. Two types of fragmented objects were depicted in the study. In the first picture a familiar object was fragmented; the second object was rendered meaningless by fragment rotation or displacement. Even when subjects could not name the objects, they reported the correct word well above chance among four presented match options in the meaningful condition.

These experiments demonstrate verbal-verbal and verbal-nonverbal semantic priming in the nonverbal analogues of the RAT as well as evidence of dual hemispheric processes operative in intuition. This semantic priming of intuitive hunches was also replicated for a study of performance on insight vignettes. ${ }^{45}$ In this study, the graphical representation of the problem space focused on implicit concepts for the insight solver group and conventional meanings of words for the noninsight solver groups. Thus, multiple lines of evidence support the notion that intuition precedes actual insight, through utilization of subconscious processes. ${ }^{43}$ However by what mechanism is this accomplished? It appears that, subthreshold activation of separable knowledge structures, eventuates in convergence on word meanings and is an example of semantic memory priming effects. ${ }^{43}$ Hence verbal-verbal (eg, remote associates test) and nonverbal-verbal (eg, fragmented picture and object naming) experiments both illustrate subconscious semantic priming. A perceptual transformation of components, borrowing heavily from Biederman's recognition by components theory of object recognition, ${ }^{51}$ could account for insight problem solving through intuitive processing within the nonverbal domain. ${ }^{52}$ There are plausible neural mechanisms to support verbal-nonverbal semantic priming. Tarr found bilateral occipitotemporal activation in subjects' decision-making involving nonsensical objects, all constructed with similar categorical features. Mental imagery generation also relied on the perceptual occipitotemporal cortex used in such basic object recognition processes. ${ }^{53}$

Hence, the essential link between intuition and insight is incubation. Incubation has been demonstrated to occur only after periods of delay, ${ }^{54}$ and ability was confirmed to interact with incubation performance effects. This is 
congruent with the hypothesis that the ability of insightful individuals to piece together the elements of a solution to a problem correlates with their ability to form a confluence of intuitive ideas through incubation. The finding relates directly to our atypical subject hypothesis, to be described below. It was found that only high ability students benefitted from an incubation period during a word solving problem. ${ }^{55}$ This suggests that congruent with many previous psychometric studies that there is indeed a reliable and solid correlation between high IQ or measured intelligence and the production of highly "insightful products" (eg, patents, inventions or wealth creation). ${ }^{56}$ This evidence is illustrative of an "IQ threshold" that occurs for productive incubation, in much the same way that no relationship has been demonstrated between IQ and creativity, beyond an IQ of approximately $120 .^{57}$

\section{Nonverbal decoding and intuition}

Lieberman provides a comprehensive framework for understanding what intuition is neuropsychologically, and perhaps more importantly, what it is not. ${ }^{30}$ Some of Lieberman's discussions are relevant to the hypotheses examined in this review. Lieberman suggests that intuition is fundamentally the "...subjective experience associated with the use of knowledge gained through implicit learning... (p. 109)." ${ }^{30}$ He directly links intuitive processes with implicit learning mechanisms. Damasio similarly notes that intuition results from cumulative affective reaction(s), rather than the deliberative cost-benefit analysis characteristic of conscious analytical processes.$^{58}$ Lieberman further elaborates the longheld view that intuition is closely aligned to social judgment processes, ${ }^{59}$ and more relevant to neuropsychology - that it is fundamentally related to nonverbal decoding. "...In the domain of nonverbal decoding, cue sequences are used to gauge the emotions, personality, intentions, attitudes or skill levels of others... (p. 111)." 30

In manuscripts discussing intuition since Lieberman, if a commonality is to be found, it is that nonverbal decoding is at the core of a comprehensive understanding of this difficult concept. ${ }^{30}$ Nonverbal subtexts underlining communication are numerable; a nonexhaustive list includes: (i) sequencing (ii) facial expressions (iii) prosody (iv) face value skill assessments of others (v) vocal expressions (vi) imitative behaviors (vii) fluency (viii) fluidity (ix) timing (x) tone of voice (xi) blinking (xii) posture (xiii) meaningful and meaningless gesture interpretation and (xiv) gaze direction. ${ }^{30}$ Many, nonverbal decoding skills, have been found to rely heavily on right hemisphere networks and substrates. ${ }^{60,61}$
Similarly, through utilization of modern neuroimaging methods, it was discovered that decision-making about the agentic qualities of body parts and "point light walkers in which the moving joints are illuminated", consistently activated the dominant inferior parietal cortex. ${ }^{62-71}$

Nonverbal decoding is extremely pervasive and is the primary means of feedback through which human beings "observe" the mental states of others. ${ }^{72}$ Nonverbal behaviors are often more accessible to the people who observe them than the actual producers of such actions. ${ }^{73}$ As human beings we are constantly and involuntarily communicating rich information pertaining to our emotional states, our attitudes, and evaluations of situations. Encoding, then, refers to the translation of a mental state into behavioral observables similar to those listed above under nonverbal subtexts to communication. It is becoming increasingly clear that, for the most part, individuals are unaware of their own emotional encoding into nonverbal observable behaviors. ${ }^{74}$ In fact, intentional and deliberative management of one's interpersonal impressions through nonverbal behavior often lead to substandard results compared with the use of intuitive social nonverbal cueing alone. ${ }^{75}$ These findings support the existence of a capacity-limited, modular nonverbal encoding neural network, with critical sequencing capacity, which is separable from the declarative memory system.

In his highly influential review, Lieberman suggested five reasons for the similarity between nonverbal decoding and implicit learning. ${ }^{30}$ These include the fact that both involve: (i) temporal sequencing and prediction (ii) learning that occurs outside of awareness (iii) the generation of representations and motor skills that are applied absent of awareness (iv) division into judgment and action skill components and (v) dependence on multiple trial learning. Implicit learning figures prominently in these nonverbal decoding experimental paradigms then. However, such paradigms are difficult to explain without actually describing the procedures that are involved and Markovian grammars are a good example. If covariation and frequency detection are at the core of implicit learning, then knowledge of Markovian grammars should provide a case in point of what is actually absorbed at an abstract level in intuition. ${ }^{76}$ Markovian grammars are based on a complex set of sequencing rules, motor skill learning, and classification judgments. Hence participants learn probabilistic contingencies among predictors, despite the impossibility of conscious analysis.

For example, on a test trial participants are asked to classify a sequence as grammatical or nongrammatical. 
Although participants perform at above chance, they report use of intuitive guesswork rather than that of real declarative information. ${ }^{77}$ Further studies have discovered that the deep structure of a grammar can transfer to a completely new set of pictorial icons ${ }^{78}$ or even across sensory modalities. ${ }^{79}$ These simple experiments suggest that intuition has adapted over the course of evolutionary history, to be sensitive to highly invariant, sequential, and causal relationships among variables in the external environment, particularly with respect to cross-modal sensory integration in the nonverbal behavior domain. However, missing until recently, were comprehensive neuropsychological models of the specific neural substrates involved in implicit learning, and hence, also in intuition (Figure 2).

The basal ganglia degenerative dementias are illustrative of diseases that may be used to understand implicit learning and hence intuition's neural substrates. Parkinson's disease is one such model utilized to gain understanding of sequence learning and basal ganglia functioning; since the putamen is affected earlier than the caudate. ${ }^{80}$ Moreover, the putamen is connected to motor areas of the cortex, whereas the caudate nuclei are connected to the dorsolateral, orbitofrontal, and anterior cingulate regions involved respectively in working memory; emotion and inhibition; and attentional shifting. ${ }^{81}$ This division, along the striatum, led Lieberman to hypothesize that the putamen is involved primarily in nonverbal encoding intuitive social action (eg, production). In contrast, the caudate has a greater role in the nonverbal decoding essential for intuitive social cognitive comprehension. Similarly, Huntington's disease results from initial destruction of the caudate.$^{82}$ Incidentally this atrophy correlates with worsening performance on the Weschler Adult Intelligence Scale (WAIS) and the Wechsler Memory Scale (WMS) ${ }^{83}$ Moreover, frontal patients performed normally on probability classification tasks, whereas Huntington's patients were impaired. ${ }^{84}$ This implies that the caudate is a seat of higher cognition.

Nonconscious processing and predictive sequencing also appear to be essential functions performed by the basal ganglia. ${ }^{30}$ Skills initially begin as conscious and deliberative, and involve the prefrontal cortex and particularly the medial aspect of temporal lobe. ${ }^{85}$ Gradually, with routinization and automaticity, these skills become completely under the control of basal ganglia structures including the striatum, ${ }^{86}$ in conjunction with the cerebellum. This also occurs for semantic types of tasks having any type of motor component. ${ }^{87}$ As temporal cues involved in sequence learning become increasingly automatized, progressive temporal pattern completion processes results in individuals being unable to determine which early cues could be guiding an intuition. ${ }^{88}$ This explains the sublime difficulty of delineating where, when, and how a particular intuition first arose since it progressively becomes automatic and under the control of subcortical motor structures. Intuition is then a property of motor sequencing of nonverbal cues (Figure 3 ). When pattern completion occurs, the remainder of a sequence can be activated resulting in an overall gestalt emerging from partial data. ${ }^{89}$ The pattern completion property of the basal ganglia may explain anecdotal evidence of intuition's capacity to even predict future behaviors or seemingly inanimate events happening in the distal environment!

Predictive sequencing is exemplified by the serial reaction time task in which subjects determine where a target will appear on a screen. The training paradigm involves the movement of the target through eight repetitive sequences. Over trials subjects implicitly learn the sequence, and during the test trial a portion of the sequence is altered. This enables the examiner to assess how well the

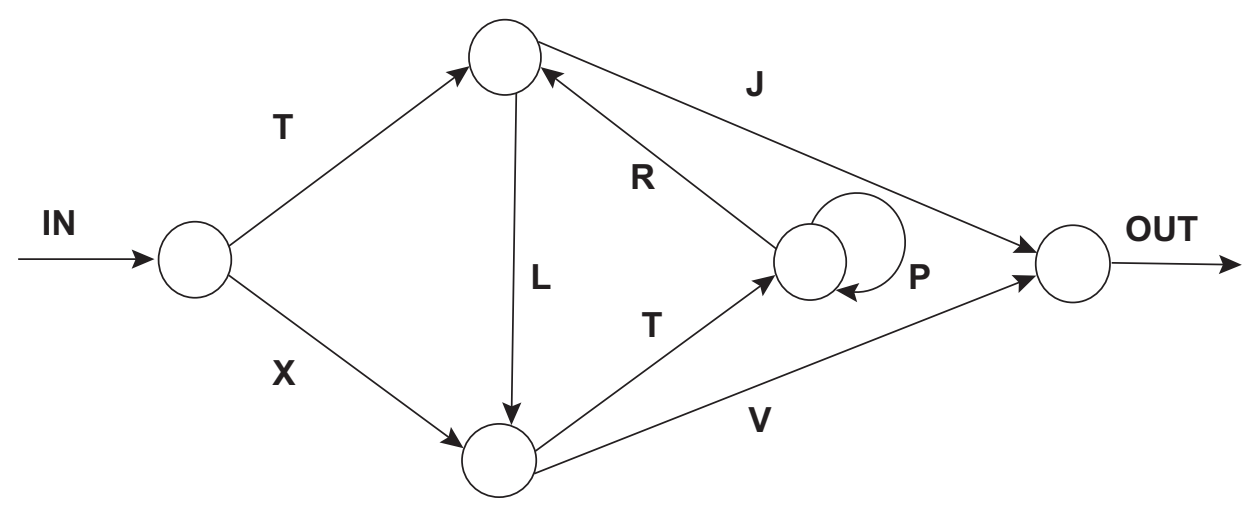

Figure 2 Example of a Markovian grammar. Grammatical strings include XV;TLV;TLTPPRj; XTRLTRJ. Copyright @ 2000, American Psychological Association. Adapted from Lieberman MD. Intuition: A social cognitive neuroscience approach. Psychol Bull. 2000; 126: 109-137. 


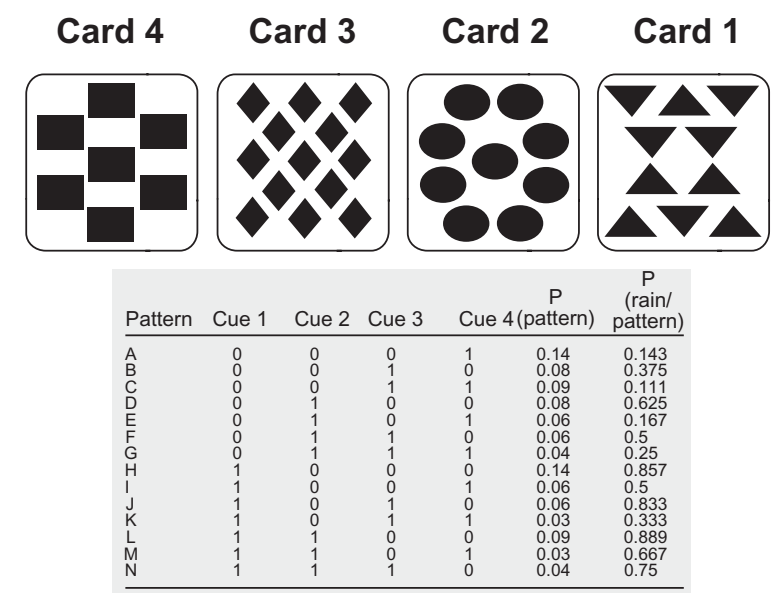

Figure 3 In the top picture, the four cards used as cues in the Weather Prediction task are depicted. Each card was associated with each possible outcome with a fixed probability. In the table is the probability structure of the task. For each pattern, each card could be present (I) or absent (0). The all-present (IIII) and all-absent (0000) patterns were never used. The overall probability of rain, given by summing $\mathrm{P}$ (Pattern) *P (rain/pattern) for all patterns, is $50 \%$. Please note that the weather prediction task is not at all like a "video-gambling task" since there is a highly complex rule-governed underlying pattern of sequences that can be used to optimize performance much like natural patterns in the external world. Copyright (c) 2002, Cold Spring Harbor Laboratory Press. Gluck MA, Shohamy D, Myers C. How do people solve the 'weather prediction' task?: Individual variability in strategies for probabilistic category learning. Learn Memory. 2002;9:408-4I8.

sequence was learned. Early Parkinson's disease patients were found to be impaired on the serial reaction time task, ${ }^{90}$ as were Huntington's patients, ${ }^{91}$ while amnesics were found completely unimpaired. ${ }^{92}$ Of significant interest was the finding that Parkinson patients' performance was unimpaired on the task when subjects were directly taught the sequence. ${ }^{93}$ The finding implies that nonconscious functions and those associated with predictive sequencing are localized in the striatum. Analogously, caudate activation was recorded during probabilistic classification (eg, the weather prediction task), which did not involve motor response. This illustrates the difference between putamen-motor and caudate-cognitive network attributes. ${ }^{94}$ Using Markovian grammars ${ }^{76}$ Lieberman and colleagues found that the right caudate nucleus was activated in response to generation of abstract rules of the grammars. ${ }^{95}$ Whether there is differential hemispheric caudate activity in other intuitive tasks is a matter for empirical inquiry. Moreover, functional connectivity analyses showed that the right caudate was negatively correlated with activation in the right hippocampus only $(r=-0.87)$ suggestive of hemispheric and lateralized intuitive grammar abstraction processes. ${ }^{95}$ Hence, these cognitive nonverbal sequencing tasks are elementally similar to nonverbal decoding functions of the right inferior hemisphere tracts and provide an experimental verification of intuition's close fit with implicit learning paradigms (Figure 4).

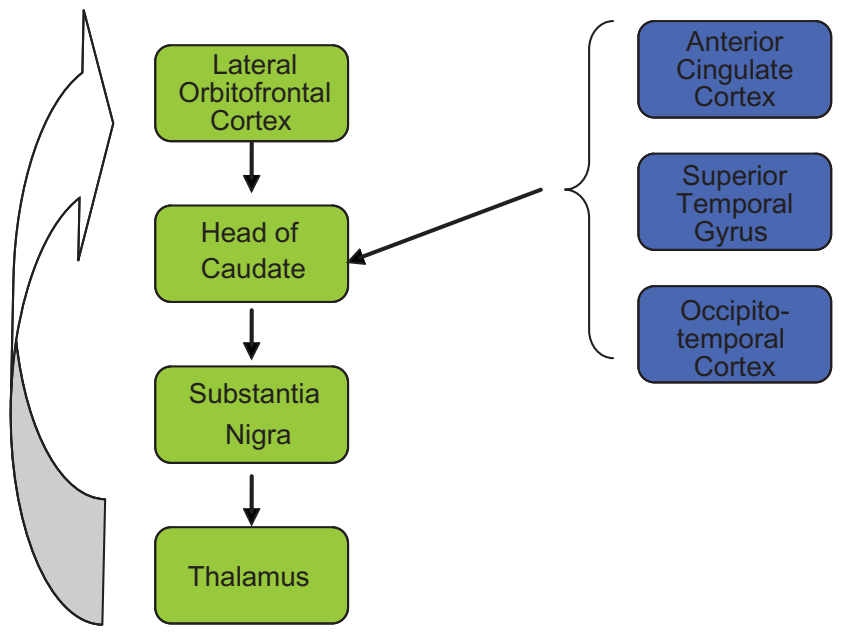

Figure 4 The parallel organization of functionally segregrated circuits linking the basal ganglia and cortex. The orbitofrontal cortex and basal ganglia are primary constituents of Lieberman's automatic X-system associated with the skilled performance and expertise used in intuitive processes. The ventral semantic stream twists through the basal ganglia's external capsule linking the posterior occipitotemporal cortex with the lateral prefrontal cortex associated with the reflective C-system. Specifically, the right anterior superior temporal gyrus is uniquely associated with insight and the caudate is associated with intuition. In this model the caudate nucleus provides the primary means by which these basal ganglia-dependent (green) and cortical systems (blue) can interact and effectively influence each other. Copyright (C) 1986. Alexander GE, DeLong MR, Strick PL. Parallel organization of functionally segregated circuits linking basal ganglia and cortex. Annu Rev Neurosci. 1986;9:357-38।.

\section{Intuition is primary and insight is secondary}

Feeling-of-knowing is perhaps one of the most elaborate accounts of the relationship between objective and subjective indices of knowing. ${ }^{96}$ This concept is exemplified by the tip-of-the-tongue phenomenon and perhaps illustrates the temporal sequence of intuition and insight. Feeling-of-knowing is usually conceived of as consisting of metacognitive judgments of ease of processing, ${ }^{97}$ fluency, ${ }^{98}$ or subjective confidence. ${ }^{99}$ Intuition is characterized: ${ }^{47}$ by (i) a dim awareness of the processes involved (ii) fast and effortless nature (iii) independence from intention (iv) generation of internal somatic cues such that these processes function to integrate vast amounts of complex information. ${ }^{100}$ In a series of 11 elegant experiments it was shown that high fluency gave rise to brief positive fluctuations in affect that emerged into consciousness as a feeling of ease in making coherence judgments of either word triads, visual images, or Markovian grammars. ${ }^{47}$ In these studies subjects completed the verbal or image based coherence judgment under time constraints such that explicit verbal answers to the problem had not yet been arrived at - yet a strong feeling of knowing guided performance on the tasks (Figure 5).

Word triads ${ }^{46}$ usually attributed to bilateral or right hemisphere functioning ${ }^{10}$ were used in the Topolinski and 


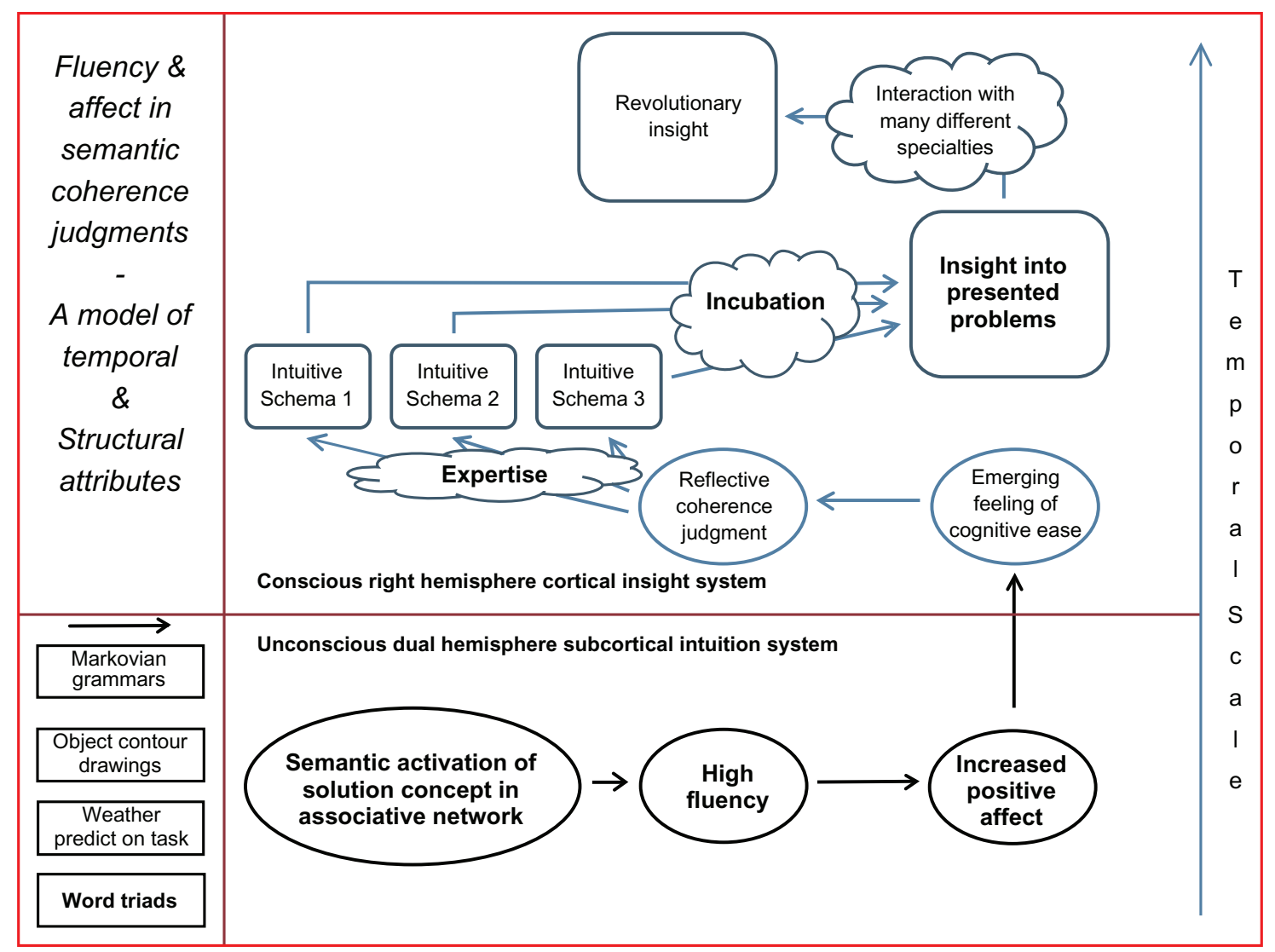

Figure 5 Although research suggests that presented insight problems do indeed rely on more lateralized right hemispheric processes - the question as to whether longer-term revolutionary insights do as well is open to conjecture and is purely speculative. However, the convergent theme that right hemispheric dominance could accentuate the development of revolutionary insights is borne out by the analysis of atypical subjects and our discussions of three of the most influential intellectuals of the 20th century. For example, Freud developed his theory of the unconscious through uncanny observations about socioemotional functions in his patients and biographers have questioned question whether Einstein had some form of developmental dyslexia (eg, see Heilman for discussions of deep dyslexia). ${ }^{17}$ Finally, Darwin developed his theory of natural selection through the integration of huge volumes of information derived from his drawings of different species.Another timely and interesting trend was that this year alone in 2009 five of thirteen new Nobel Laureates were women, the largest number to ever join the ranks in a single year. Copyright @ 2009 . Adapted and redrawn from Topolinski S, Strack F. The architecture of intuition: Fluency and affect determine intuitive judgments of semantic and visual coherence and judgments of grammaticality in artificial grammar learning. J Exp Psychol Gen. 2009;138:39-63.

Strack study. ${ }^{47}$ Previous studies have shown that reading of the words is usually processed in the left hemisphere; ${ }^{101}$ whereas distant or "coarse" concept mapping between words may be the prerogative of the right hemisphere - even in subjects that are not right hemisphere dominant. ${ }^{2}$ Judgments of visual coherence in fragmented object contour drawings was taken from the Snodgrass and Vanderwart collection. ${ }^{102}$ Processing of whole pictures and visual images is usually considered a property of the integral functioning of the right hemisphere ${ }^{103}$ Hence there is some good evidence to suggest that intuition is content specific involving verbal and nonverbal material and yet that these processes are still temporally and structurally the same. ${ }^{47}$ Since these studies used the same archetypal task that is used in insight studies namely the word triads, and generalized these findings to other tasks (object contour drawings and Markovian grammars) 48,104-106 $^{-10}$ this illustrates how intuition is primary. This last point is all the more true since the archetypal remote associates task is entirely consistent with more real-world insight tasks. ${ }^{107}$

\section{Emotion, affect, and mood in intuition?}

The first stage of stimulus emotional appraisal is the distinction between positive and negative affective valence. ${ }^{108}$ The basal ganglia appear involved in this initial assessment of positive stimuli; ${ }^{30}$ since left basal ganglia lesions reliably result in depression, ${ }^{109}$ and because both Parkinson's and Huntington's disease ${ }^{110}$ often are associated with organic depression. Unipolar depressive disorder is associated with decreased metabolism in the basal ganglia, ${ }^{111}$ as well as with significantly increased serotonin binding potential (indicative of decreased serotoninergic tone during episodes), within the striatum. ${ }^{112}$ In contrast the amygdala controls bodily responses to aversive stimuli and plays a central role in responding to 
fear-inducing and negatively-valenced stimuli. ${ }^{113}$ Arguably, the basal ganglia (particularly the striatum) and the amygdala comprise two essential way-stations to the positive and negative affective stimulus appraisals typical of the intuitive social cognition system.

Interestingly, female superiority in terms of nonverbal behavior decoding skill ${ }^{114}$ and an estrogen-mediated striatal increased performance function on sequential learning tasks, ${ }^{115}$ provide empirical and anecdotal corroboration of a biologically based female advantage on many complex intuitive social cognition tasks. ${ }^{30}$ These female advantages seem to be manifest in enhanced cortical-subcortical connectivity. Individual differences in the subcortical transfer of semanticmotor information has been hypothesized for some time. ${ }^{116}$ In a study of commissural transfer it was found that those with strong leftward hemisphere dominance tended to have smaller areas within the midbody of the callosum. ${ }^{117}$ However there is good evidence of neural plasticity and canalization of function through the subcortical and cortical commissures. Marzi and colleagues showed that visuomotor interhemispheric transfer takes place through the parietal commissures in normals, and through the anterior or subcortical commissures in a commissurotomy subject using positron emission tomography. ${ }^{118}$ Collectively, then, gender differences in both interhemispheric, and perhaps more provocatively, intrahemispheric subcortical-cortical connectivity, could be worthy of future intuition and insight studies.

At the level of analysis of nonverbal decoding differences, and in the context of social psychology, there are several implications for such individual and group differences. This is illustrated by two well-known theories of social cognition and attitude change. ${ }^{19,120}$ At the time both theories were heavily reliant on a declarative memory system where it was assumed that declarative memory for counter-attitudinal behavior was essential for behavior-induced attitude change. ${ }^{30}$ However, a recent study of attitude change in anterograde amnesics found contrarily that these subjects showed as much attitude change as did control participants. ${ }^{121}$ Similarly, a reflected appraisal or "what she thinks he thinks of her" resulted in activation of an entirely right hemispheric network, including the ventrolateral prefrontal and medial temporal cortex, medial parietal lobe, when compared with self-directed appraisal. ${ }^{122}$

A right hemisphere based nonverbal decoding advantage that facilitates female intuitive judgments is consistent with all of the above hypotheses. In addition, if the right hemisphere network runs anteriorly and ventrally, extending from the occipitotemporal cortex to the orbital and dor- solateral prefrontal cortex, it is likely to involve the rapid conducting inferior occipitofrontal fasciculus. ${ }^{123,124}$ Clearly, cortical and noncortical substrates are involved in any contemporary model for emotional interaction in the appraisal of intuitive tasks. For instance, re-appraisal of a strategy for emotional self-control or self-regulation, in which subjects were required to decrease and control negative affect, involved activation of the right lateral orbitofrontal cortex implicated in behavioral inhibition. ${ }^{125}$ Similarly, studies have shown that unintentional affect labeling, resulted in increased activity in the right ventrolateral prefrontal cortex, with a resultant inverse relationship between activity in the prefrontal area and activity within the amygdala. ${ }^{126}$

\section{Neural correlates of intuition}

Lesion studies are not the only means of examining the neural correlates of right hemisphere based nonverbal decoding. One conceptualization of intuition is that it results from the integration of affectively laden gists with unconsciously represented decision-making information. Lesions of the orbitofrontal cortex result in reliable deficiencies in decision-making of this type. ${ }^{127}$ Goal valuation is associated with neural activity in the medial orbitofrontal cortex, whereas decision valuation is associated with activity in the central orbitofrontal cortex, and prediction error signaling is associated with reliable activity in the ventral striatum. ${ }^{128}$ Hence, orbitofrontally lesioned patients were found unable to integrate emotional and visceral signals into decisionmaking scenarios. ${ }^{27}$ The orbitofrontal ventral neural network hypothesis of intuition and insight will be developed further in a subsequent section.

In one study, participants were presented with fragmented black-and-white line drawings of common objects ${ }^{26}$ taken from Snodgrass and Vanderwart's collection. ${ }^{102}$ The task was to determine if the object was meaningful (real object) or nonmeaningful (nonobject). Subjects were asked not to name the object, but rather to rely on their gut feeling about whether the pictured stimulus depicted a coherent gestalt. The investigators found activation in the medial orbitofrontal cortex when a fragmented drawing was judged a coherent gestalt. When subjects were asked to individuate objects in a neuroimaging study it was found left orbitofrontal cortex activity $50 \mathrm{msec}$ earlier than activity recorded in the fusiform gyrus. ${ }^{124}$ These findings suggest that low frequency information in such drawings facilitates object recognition by initiating top-down processes projected from orbitofrontal to visual cortex. It is possible that a similar right-sided orbitofrontal network exists in right hemisphere dominant individuals. 
Again, from a functional neuroanatomical perspective there are specific hypotheses about what white matter tracts would transfer such information. It was proposed that the information being transferred from the orbitofrontal cortex $^{26}$ to the inferior temporal cortex utilizes the inferior occipitofrontal fasciculus. ${ }^{129}$ By way of digression the ventral occipitotemporal cortex consists of the fusiform gyrus laterally to the parahippocampal gyrus medially; activation in the ventral occipitotemporal cortex was suggested to represent holistic object perception processes. However, it is possible that another route, other than the inferior occipitofrontal fasciculus, conveys part or all of this information. A second possibility would be the inferior longitudinal fasciculus connecting the occipital and anterior temporal lobes, in tandem with the uncinate fasciculus connection from the anterior temporal lobe on to the orbitofrontal cortex. However, it is suggested that this second $\mathrm{U}$-shaped fiber two-way route would be incapable of the required less than $50 \mathrm{msec}$ neural transmission ${ }^{26}$ shown to be necessary in Bar and colleagues's magnetoencephalography study. ${ }^{124}$

The second two-way route described above carries visuosemantic information emanating from the temporal pole. ${ }^{130}$ Therefore, the inferior frontooccipital fasciculus is likely a primary conduit of the intuitive knowledge network. ${ }^{26}$ And functional connectivity analysis demonstrates simultaneous activation within right anterior claustrum, anterior insula, and amygdala. The claustrum is closely associated with striatal functioning and could be involved in synchronization of large-scale whole brain networks. ${ }^{131}$ This function would allow an affectively valenced percept to exhibit a characteristic judgmental sense of rightness by temporally linking intuitive processers. Such a clock-like mechanism could also account for the anecdotal findings of a fundamental aspect of intuition which is initiating the correct behavior at the optimal time. Finally, this right hemispheric network extending posteriorly from the right inferior occipitoparietal lobe all the way on to the right orbitofrontal cortex would also be congruent with findings suggesting that these structures constitute a "when or optimal timing pathway" for stimuli within both visual fields. ${ }^{132}$

\section{Neural correlates of insight}

Intuition, then, is associated with ventral mixed corticalsubcortical interconnected networks extending from the most anterior and posterior reaches of the cerebral cortex. These building blocks of intuition are involved in automatic, nonconscious, and skilled nonverbal decoding especially associated with the right posterior parietal regions. Insight, by contrast, is a conscious and deliberative process at least past the point of restructuring. Recall that restructuring is the rotation of multiple concepts in memory until a semantic gestalt is arrived at that seems logical from any vantage point. Ilg and colleagues used a unique design ${ }^{133}$ in which aspects of the $\mathrm{RAT}^{46}$ were modified so that subjects were required to provide speedy responses. Recall that RAT problems consist of a word triad such as (pine, crab, sauce) and the subject attempts to produce a single solution word (apple). The solution word can form a familiar compound word or phrase with each of the three problem words (pineapple, crab apple, applesauce). RAT and classic insight problems are similar in that these short items (i) misdirect retrieval (ii) solving processes are nonreportable and (iii) elicit aha or eureka responses. ${ }^{134}$

These word triads are not as complex as classic insight problems and have advantages of being able to be: (i) solved in short period of time; (ii) have single unambiguous word answers; and are (iii) physically compact for presentation in functional neuroimaging environments. Finally, and most importantly, these "mini-insight" problems are highly correlated with lengthy verbal insight problems. ${ }^{23,107}$ Therefore given the speeded response in the Ilg study only a subconscious intuitive coherence judgment could be elicited and not an explicit judgment. Recall that the RAT is the abbreviated insight problem of choice where the 'eureka' or 'aha' experience can be tested for. ${ }^{10}$ The condition of explicit judgment (eg, the participants actually picked out the correct RAT) was subtracted from those who made an implicit judgment of coherence condition (eg, an implicit judgment that yes the three words were coherent but that the solution was not conscious). In this intuitive experimental condition, reliable activations were found in the heteromodal bilateral inferior parietal cortex and the right superior posterior temporal cortex. ${ }^{133}$

The results of this experiment were unambiguous and confirmed that intuition and insight are related entities. In contrast, increased activation in the anterior right superior temporal cortex was found during sudden insight, ${ }^{135}$ using either electroencephalography (EEG) or fMRI. In an insight EEG study, a negative correlation between subjective ratings of the problem restructuring process and the feeling of suddenness of the solution, suggested that restructuring involves an automatic, subconscious recombination of information. ${ }^{136-138}$ Similarly, the researchers found increased upper alpha band response in right temporal regions, that was interpreted as suppression of weakly activated solution information. ${ }^{137}$ Mental impasse was associated with activation in parietooccipital regions in the gamma band and was hypothesized to be associated with inappropriate problem representation and an overload of selective attention. ${ }^{139}$ Finally, in trials 
with high restructuring, decreased alpha power indicative of greater cortical excitation, was discovered in the right prefrontal cortex. Tellingly, both lesion ${ }^{140}$ and $\mathrm{fMRI}^{141}$ studies similarly have shown that restructuring of insight problems involves the right prefrontal cortex as well.

Right hemisphere biases in processing occur when problem solver's aha experiences are associated with insight solutions and especially with subconscious semantic priming. ${ }^{142}$ However the comprehension associated with insight is not completely unilateral. Bilateral anterior superior temporal gyral neural activity has been found to correlate with comprehension of sentences and complex discourse $\mathrm{e}^{143,144}$ and these areas are probably recruited during the initial setting of the parameters of a verbal insight problem. Also signal originating in the right anterior superior temporal gyrus during insight problem solving was not due to emotion associated with "aha" because it only increased when subjects first encountered this problem. ${ }^{135}$ Revealingly, right hemisphere coarse coding may not be restricted to only nouns since generating unusual verbs to nouns resulted in activity in the right frontal gyri, ${ }^{145}$ and comprehending extended discourse resulted in activity in right frontal lobe. ${ }^{146}$

This importance of the right frontal cortex in restructuring, associated with insight problem-solving is noteworthy. Frith suggested that of the myriad functions of the prefrontal cortex, 'sculpting of the response space' subsumed them all. ${ }^{147}$ That is the dorsal lateral prefrontal cortex (DLPFC) is crucial for defining a set of responses, suitable for a particular task, and then biasing these for selection. The matchstick insight problem is another example of a complex insight problem where configurations of matches must be used to solve Roman Numeral type diagrams with explicit rule-based constraints. The matchstick insight arithmetic task is biased by strong constraints, that prevent participants from considering and evaluating the correct solution; ${ }^{148}$ and eye movement studies support that problem restructuring occurs by constraint relaxation and chunk decomposition. ${ }^{24}$ That is one set of constraints or rules are ignored so that other essential features of the problem can be attended to in sequence (constraint relaxation) and/or chunks of the problem are decomposed into more manageable units (chunk decomposition).

The DLPFC therefore excludes the correct interpretation since normals solved only $43 \%$ of the most difficult matchstick problems; whereas $82 \%$ of DLPFC patients did. ${ }^{149}$ Carlo Reverberi noted this effect was stronger in right DLPFC (pers comm, Sept 2008). Hence, the right DLPFC could have an essential role in constraining the response space of insight problems. This fits assumptions about real world scenarios where preliminary downgrading of all potential possibilities is essential in order to gain traction on a complex insight problem. Hence, a number of generalizations about the functional relationships of intuition and insight include: (i) intuition is associated with nonverbal decoding (ii) intuition is associated with implicit and reflexive processes (iii) insight is associated with conscious restructuring processes (iv) intuition feeds into insight based right hemisphere networks through the right caudate ${ }^{95}$ and is modulated by the orbitofrontal cortex.$^{81}$ In a very real sense then intuition is primary and insight is only a secondary process that is reliant on the expertise and automaticity of intuition.

\section{Conscious and unconscious processes}

If we are discussing the right hemisphere, then surely any examination of superior social cognition constructs like intuition and insight must elaborate on how these processes relate to the weighty topic of the "self". It is difficult to imagine concepts like intuition and insight, that that are so lawfully and obviously related to the construct of consciousness, would not also have empirical and theoretical relationships with consciousness's object, the self. Indeed, many studies using structural, functional neuroimaging, and lesion methods have definitively localized salient aspects of the "self" to the right hemisphere. ${ }^{150}$ The self usually is, after all, associated both in common parlance, and within neuropsychological theory, with the right hemisphere's emotion and gnostic functions. ${ }^{61}$ The UCLA social cognitive neuroscience laboratory similarly has made progress on hypotheses concerning the self's relationship to intuition, and to a lesser extent insight. In Lieberman and colleagues' terminology, the C-system (an abbreviation for the $\mathrm{C}$ in refleCtive), is implicated in intentional social cognition, which takes effort and is reliant on propositionally organized symbolic representations that can be processed serially, in working and episodic memory. ${ }^{151}$ That episodic and working memory function together in the $\mathrm{C}$-system is evidenced in levels of processing research which concludes that greater working memory demand at encoding, results in superior episodic retrieval success. ${ }^{152}$ Neural correlates of the C-system include the lateral prefrontal cortex, posterior parietal cortex, hippocampus and the medial temporal lobe structures. ${ }^{153}$ Hence, C-system structures are used for evidence-based self knowledge, similar to those associated with propositional reasoning ${ }^{154}$ (Figure 6).

A series of ingenuous experiments revealed that the $\mathrm{X}$ - and $\mathrm{C}$-systems have definitive relationships with the 


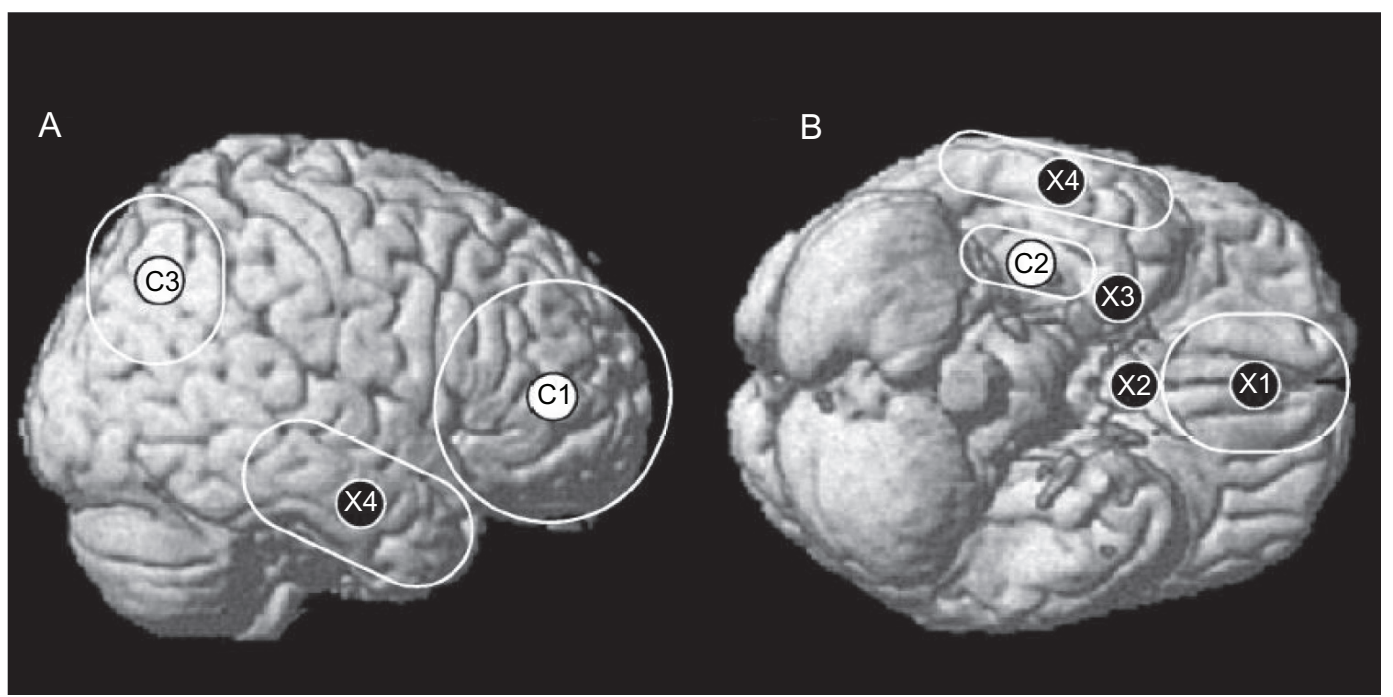

Figure 6 Neural correlates of the C-system and the X-system displayed on a canonical brain rendering from side (A) and bottom (B) views. C-system regions displayed are the lateral prefrontal cortex $(\mathrm{Cl})$, hippocampus and medial temporal lobe $(\mathrm{C} 2)$, and the posterior parietal cortex (C3). $\mathrm{X}$-system regions displayed are the ventromedial prefrontal cortex $(X 1)$, nucleus accumbens of the basal ganglia (X2), amygdala (X3) and the lateral temporal cortex (X4). Please note that the hippocampus, nucleus accumbens and amygdala are displayed on the cortical surface for the sake of clarity. Copyright () 2004, American Psychological Association. Adapted from Lieberman MD, Jarcho JM, Satpute AB. Evidence-based and intuition-based self-knowledge: An fMRI study. J Pers Soc Psychol. 2004;87:42 I-435.

concept of the self. When participants made self-judgments in domains in which they had little experience, there was strong evidence for the use of episodic retrieval. ${ }^{155}$ Therefore, evidence-based self knowledge is not the only means of self-judgment, and it may be limited to those low experience domains of knowledge that subjects have about themselves. In other words if subjects make decisions about themselves in a domain that they have little knowledge they will more often than not use the reflective $\mathrm{C}$-system. In contrast, if subjects make decisions about themselves in domains that they tend to be experts in they will use the X-system. A provocative example is that of patient $\mathrm{DB}$ who was permanently amnesic for his entire life, yet was able to make accurate self-referential personality ratings. ${ }^{156}$

Thus the $\mathrm{X}$-system (as the $\mathrm{X}$ is used in refleXive), automatically collates social and affective variables and produces habitual responses. ${ }^{151}$ The X-system consists of the ventromedial prefrontal cortex, basal ganglia, amygdala and the lateral temporal cortex. ${ }^{157}$ This research group conducted a fMRI study in which soccer players or actors made self-judgments in a high-experience or low-experience domain respectively. ${ }^{154}$ The purpose of the study was to separate the neural substrates of the volitional C-system and the automatic X-system. Subjects made decisions on whether actor or athlete words described themselves accurately. The results showed that self-descriptive judgments from high and low-experience domains were the same for both groups. This finding suggested generalizability across expertise domains and an index of schematicity was computed. The index of schematicity reflected the degree of schema strength in the high-experience domain relative to the low-experience domain. It was predicted that higher difference scores reflected the presence of a more developed schema in the high experience domain. ${ }^{154}$ Regions found more active in the intuition-based selfknowledge judgments, associated with the X-system's high-experience domain, included the left ventral frontal cortex, the left nucleus accumbens of the basal ganglia, the left amygdala, the right lateral temporal cortex and the right posterior parietal cortex. In contrast, the only regions activated with the $\mathrm{C}$-system in nonschematics included the right hippocampus, the bilateral precuneus, and the bilateral dorsolateral prefrontal cortex. ${ }^{154}$ The ventromedial prefrontal cortex was uniquely activated by the $\mathrm{X}$-system and the hippocampus was uniquely activated by the C-system such that when subjects made judgments in their high-experience domain, they elicited activation in a network of regions not normally associated with episodic recall or explicit evaluation. However, when assessments were made in their low experience domain, only the lateral prefrontal cortex was differentially activated. ${ }^{154}$

Similarly, implicit learning is associated with the unintentional learning of probabilistic relationships between various cues. ${ }^{158}$ The increased activity in the basal ganglia and orbitofrontal cortex during intuited self-knowledge are highly consistent with an implicit/explicit memory 
dichotomy. Ventromedial prefrontal cortex activation was recorded for the implicit association test, ${ }^{159}$ providing support for the automaticity-hierarchical delineation. Finally, when nonschematics made intuitive judgments, reliable activation was recorded in the lateral temporal cortex, which has been associated with semantic memory functions. ${ }^{160}$ In sum, partial support was found for a combination of implicit/explicit, automatic-controlled processes and episodic/semantic memory involved in intuitive-like functions. However, event-related designs likely would be more helpful in determining what aspects of the neural systems are used in intuition. Hence, the functional neuroanatomy of the intuitive networks implies that the X-system is: (i) affective (ii) slow to form (iii) resistant to and slow to change (iv) insensitive to one's thoughts about one's own self and finally (v) insensitive to explicit declarative feedback from others. ${ }^{154}$ There is good evidence to suggest that the $\mathrm{C}$-system is relied on only by default in novel decisionmaking (such as novel insight problems), when heuristic automatic judgments based on associative and intuitive systems are unreliable.

Moreover, the main purpose of the [C-system] is to monitor the [X-system] decisions and correct them when wrong. ${ }^{161}$ Other studies suggest that another function of the intuitive ventral frontal and basal ganglia driven X-system is to usurp effortful, analytical processes over time so that comparable automatization benefits can be achieved with minimal efforts. ${ }^{162}$ Usurping of cortical functions by basal ganglia subcortical structures is congruent with the known functions of these structures and with previously reviewed striatal skill acquiescence. Congruently consumption of glucose restored effortful C-system functions on a decisionmaking task following completion of a dual-task paradigm. ${ }^{163}$ Thus tired subjects tended to rely on heuristic, automatic decision making strategy, characteristic of the X-system. The caudate of the basal ganglia thus acts like a way-station that directs cognitive activity along automatic default processing networks unless novel stimuli require frontotemporal evaluation of conscious analytical signals processed for affective valence in orbitofrontal cortex. ${ }^{124}$

\section{A right hemisphere ventral network in intuition and insight?}

An example of a pervasive type of intuitive social cognition is that of the use of scripts, and the facility with which one uses these tools determines in part one's level of social competence. ${ }^{164}$ Scripts are over-learned narratives that indicate a culturally agreed upon order of events in social episodes. Scripts have a core feature of sequencing of gestalt nonverbal actions. These tools allow inference behavior that may not have been directly witnessed and thereby can be used by intuition or insight to infer mental states. Scripts are similar to structured event complexes associated with the functional social purposes of the prefrontal cortex. ${ }^{165}$ A structured event complex contains macro-level information relevant to past and current behavior through the storing of events that occurred in the past, and may be likely to occur again in the future. Each structured event complex stores both the theme and the boundaries of events assigned to it. However it does not simply re-represent words and objects stored in posterior association areas of the brain. A structured event complex enables flexible encoding of input allowing for inference and generalization and it stores events that are temporally bound together in close proximity.

A managerial knowledge unit is composed of a series of such structured event complexes and are based on: (i) sequencing (ii) flexibility in sequencing (iii) problem constraint satisfaction (iv) basal frequencies of sequences (v) durations (vi) contexts of settings for actions and (vii) goalsub-goal hierarchies. A sequence of agreed upon actions for a dinner date would be an example of a managerial knowledge unit. ${ }^{165}$ Judgments of temporal contiguity between adjacent action sequences was found to be a function of activation in the posterior association cortex, whereas goal structuring of scripts was found to be a function of the prefrontal cortex. ${ }^{166}$ Script generation versus execution errors in dysexecutive patients was consistent with a caudate, rather than a putamenal link in cognitive function. ${ }^{167}$ Likewise, the right inferior frontal gyrus was activated in a script ordering task, ${ }^{168}$ while right orbitofrontal cortex damage resulted in impairment in the accessibility of scripts, as well as in the semantic representation of social activities. ${ }^{169}$ Developmentally we can see the linking of nonverbal sequences in the right occipitotemporoparietal regions with abstractions of these nonverbal sequences in the form of scripts in the right orbitofrontal cortex.

Huntington's ${ }^{170}$ and Parkinson's patients demonstrated script sequencing deficits. ${ }^{171}$ Given the ventral loci of the right orbitofrontal script reading neural network, parallels can be drawn to a recently described "ventral semantic stream", present within the right hemisphere. ${ }^{172}$ The pathway joins the posterior and superior temporal areas with the inferior and dorsolateral prefrontal cortex, through the inferior occipitofrontal fasciculus. The fasciculus narrows at the junction of the frontal and temporal lobes and passes through the anterior floor of the external capsule of the ganglia, with terminal radiations reaching the middle and inferior temporal gyri and traveling without interruption to the lingual and fusiform 
gyri. ${ }^{123}$ We can see then that these fasiculi could possibly allow ready connectivity between posterior nonverbal decoding networks and the automatization associated with striatal networks moderated by context information within the right orbitofrontal regions.

Electrostimulation anywhere within this fasiculi resulted in semantic paraphasias. ${ }^{172}$ Semantic paraphasic errors are exemplified by labeling a presented object such as a "banana" as a "hammer". We have previously reviewed how coarse, sparse or distant semantic mappings are an intrinsic property of the right hemisphere. Given inferior occipitofrontal fasciculus proximity and connectivity to the orbitofrontal cortex, it is hypothesized that the ventral right hemispheric rapid conducting pathway links the right inferior posterior parietal nonverbal decoding regions with the rostral cognitive script interpreters associated intuition and insight. When comparing novel gesture interpretation or naturalistic action in the right inferior parietal lobes, ${ }^{173}$ with the interpretation of scripts in the right orbitofrontal cortex, ${ }^{168,169}$ there is a clear anterior-posterior demarcation in complexity of the social psychological functions. This ventral right hemisphere network appears to be essential in intuition and insight for many strong empirical cognitive science, neuropsychological, and anatomical reasons previously reviewed. Yet the true test of such a hypothesis would be to carefully examine such hypothesized right hemisphere dominant patients using structural and functional neuroimaging as well as comprehensive neuropsychological assessment. In the past the only way that such patients would be found would have been through the rare occurrence of a small localized stroke within either Broca or Wernicke's area in the right hemisphere without any attendant secondary dementing processes or confound present. These cases are exceedingly rare.

\section{Crossed aphasia from the historical perspective}

Before the era of functional neuroimaging, studies of crossed aphasia provided the only means to examine inferences regarding the nature and organization of atypical language and spatial functions when localized to the right hemisphere. In the most comprehensive meta-analytic study to date, of crossed aphasia in dextrals, Marien and colleagues found that $65 \%$ demonstrated constructional apraxia; 39\% demonstrated both constructional apraxia and visuospatial neglect; $8 \%$ showed visuospatial neglect but not constructional apraxia; and finally, 26\% demonstrated constructional apraxia without visuospatial neglect. ${ }^{174}$ This meta-analysis revealed that there is considerable heterogeneity in the type and location of different modules within the nondominant hemisphere in crossed aphasics. If there is a near random distribution in which these and other cognitive functions can be localized in one hemisphere or the other, as well as co-localized together in different combinations, then this principle provides some circumstantial support for the hypothesis of the possibility of emergent properties that are qualitatively different than individual components themselves (Figure 7).

Moreover, the incidence of constructional apraxia was greater than threefold the incidence of visuospatial neglect, and visuospatial neglect occurred at less than $10 \%$ in the sample. Since visuospatial neglect is the sine qua non of "crowding", the low incidence where there is increased lateralization of cognitive processes, can readily be interpreted as strong evidence contrary to the crowding hypothesis. Crowding suggests that with co-localization of language and spatial functions to the right hemisphere there is decrements in the performance capacities of both cognitive functions. Yet the high incidences of constructional apraxia suggests that this function often co-associates with other modular functions in these subjects. In other words, the results do not support the assertion that right-hemisphere language always is asso-

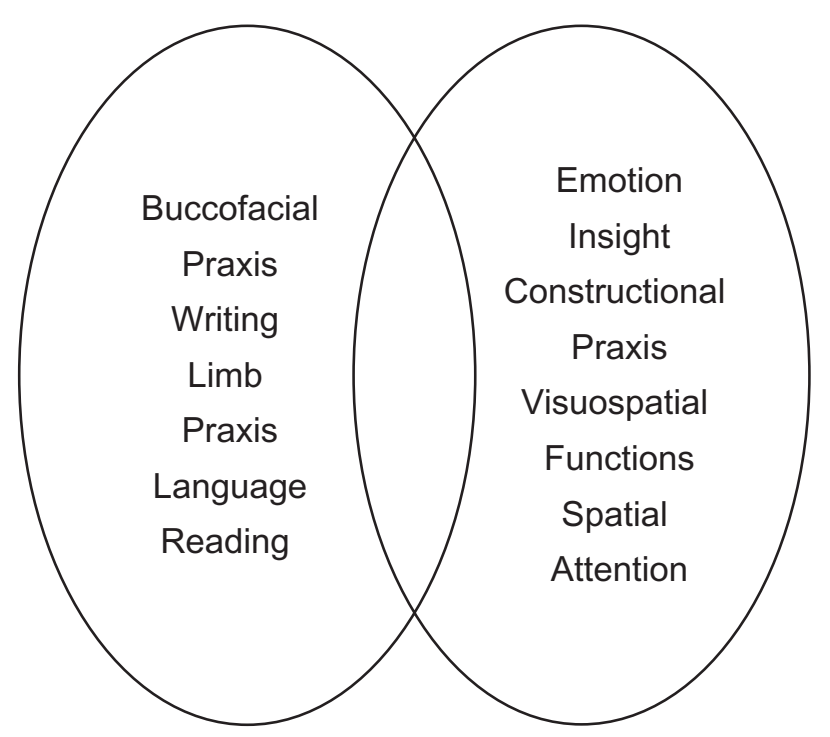

\section{Left hemisphere Right hemisphere}

Figure 7 Archetypal configuration of specific co-localized cognitive modules in normally represented lateralization of functions subjects. In Marien and colleagues'174 study of crossed aphasics with singular right hemisphere lesions a secondary analysis was undertaken. Buccofacial praxis was correlated with limb praxis $(r=0.4 I, P<0.01)$ and writing $(r=0.39, P<0.05)$. Limb praxis was correlated with writing $(r=0.67$, $P<0.001)$ and visuospatial functions $(r=0.40, P<0.05)$. Finally, visuospatial functions were correlated with constructional praxis $(r=0.35, P<0.05)$. Copyright $(\odot)$ 1991. Adapted with permission from Fischer RS, Alexander MP, Gabriel C, Gould E, Milione J. Reversed lateralization of cognitive functions in right-handers: Exceptions to classical aphasiology. Brain. | 991; | |4:245-26।. 
ciated with the crowding out of other cognitive functions, such as that of visuospatial neglect and constructional praxis. In fact, crossed aphasia in dextrals likely constitutes a highly selected sample. Indeed, Marien and colleagues note that although "... both neglect and constructional apraxia stand out as frequent nonverbal disorders in vascular crossed aphasia in dextrals, often, though not inherently, [do these symptoms] associated with each other... (p. 55)." 174 Hence, support for the crowding hypothesis, which historically was associated with the neurolinguistic hypothesis that the right hemisphere was communicatively inferior ${ }^{4}$ is not borne out by the data.

Indeed in 6 of 10 atypical language localization subjects a complete reversal of linguistic and spatial functions was found, and four alone showed co-extensive language and spatial attention processes. ${ }^{8}$ A subsequent study found that right hemispheric language dominant subjects had a greater tendency towards bilateral or leftward dominance for spatial attention suggesting developmental pressures towards complete reversal of linguistic and spatial functions. ${ }^{175}$ Some of the best evidence for the basal rates of unequivocal right hemisphere language in the general population would be found in the crossed aphasia literature pertaining to dextrals. ${ }^{174}$ Based on 152 cases published since 1975, only $32 \%$ could be reliably categorized into crossed aphasia in dextrals after exclusionary criteria such as nondextrality, unilaterality of lesion, no prior premorbidity, illiteracy, second language or presence of ideographic scripts or tonal languages were applied.

This constitutes a mere 48 known cases of verified crossed aphasia in the published literature since 1975. Interestingly, the frequency of occurrence of crossed aphasia in dextrals was compared between men and women, and men outnumbered women by nearly $3: 1$. The frequency distribution could perhaps provide clues to the inheritance mechanisms of this anomalous pattern of language representation. Specifically, in agreement with prior studies it suggests that an X-linked allele could encode the phenotype in normal healthy individuals. ${ }^{174}$ However it is possible that this ratio could be entirely a statistical artifact (and in fact very likely underestimates crossed aphasia in dextral women), since women routinely do not suffer the same degree of aphasic symptoms after unilateral stroke lesions due to the predominance of bilaterality of language localization. Secondly, when compared to men of the same age when women do have strokes that are debilitating they are usually so serious that they are rendered untestable and therefore would be naturally excluded from any such studies. ${ }^{176}$
Visuospatial neglect, the sine qua non of crowding, was found more severe in crossed aphasia in dextral women than in men! More detailed studies would be necessary to corroborate this apparent gender-linked finding. Secondly, in noncrossed aphasia there is a preponderance of nonfluent (eg, frontal) as opposed to fluent (eg, posterior) aphasics, whereas, with crossed aphasia, there is an equivalent frequency of such findings. ${ }^{174}$ Recall that noncrossed aphasia is the condition when a patient sustains a serious stroke within the left perisylvian language cortex that would be expected to result in either fluent or nonfluent aphasia yet does not - implying by default language localization to the nondominant right hemisphere. This frontality of noncrossed aphasics versus equivalent frequency of frontal or posterior crossed aphasics could be a function of the greater spatial distribution of language localization within the right hemisphere. $^{2}$ Incidentally and as expected this neuroanatomical greater spatial distribution of language processors within the right hemisphere educed from the frequency of proportions of aphasic in the crossed and noncrossed varieties agrees with the spatial summation priming and coarse coding hypothesis borne out by neuropsychological studies. ${ }^{50}$

Additionally, it was found that typology of crossed aphasia was independent of age, perhaps suggesting genetic determination for the spatial localization since older aged crossed aphasics did not happen to have more serious (eg, global aphasia) strokes. ${ }^{174}$ Again this implies that it is the nature of the spatial localization of crossed aphasia per se, rather than some intervening variable such as a pre-existent higher frequency of incidental arteriovenous malformations that is mediating the lateralized effect. Other important findings include that agraphia in crossed aphasics was found to be particularly severe pointing the way to examination of unique language and praxis localization and co-localization scenarios occurring in this population. Finally, estimates of the prevalence of the potential cases of crossed aphasia in dextrals vary. A $4 \%$ incidence of rightward language lateralization using transcranial Doppler sonography has been found, ${ }^{177}$ and $6 \%$ using fMRI. ${ }^{178}$ In the context of the exclusionary criteria this would provide a $1 \%-2 \%$ estimate of the general population as unequivocally showing strong right hemisphere dominant language in agreement with earlier surveys. ${ }^{179}$

A few points are worthy of mention in regards to crossed aphasia in dextrals and praxis localization. Again, visuospatial neglect was recorded in $43 \%$ of males and $57 \%$ of female crossed aphasia in dextral subjects. ${ }^{174}$ In contrast, either ideomotor or ideational apraxia which is associated with 
dominant hemisphere functioning, was found in $18 \%$ of male subjects and $38 \%$ of females. These findings suggest that in well-characterized subjects, less than half of crossed aphasics demonstrate the putative crowding out of other cognitive functions. This is an important point to be considered when analyzing the Marien and colleagues study since crowding is almost taken to be that of a given in conventional wisdom accepted by clinical neuropsychologists. Furthermore, the low frequency of apraxias associated with right-hemisphere subjects, suggests, that in atypicals, there are bilateral parallel distributions, perhaps more so in men or patent co-localization of praxis and language especially in women. Hence in males, praxis and language dissociation subjects may be particularly apt to demonstrate complete mirror reversal in this domain. ${ }^{180}$ There is no obvious reason as to why language and praxis would so routinely be parcellated to the opposite hemisphere in atypical or anomalous subjects!

It is likely that multiple genetic polymorphisms impact on the higher-order factor of general ability, and that studies of small population samples with rare high score IQ's, are unlikely to target specific critically involved genes. ${ }^{181}$ In this context, specific cases like that of the right-hemisphere dominant individuals discussed above, could be informative on specific genetic mechanisms involved in normal neurodevelopment, which are likely to be far more numerous and diverse than the generalist genes integral to basic cellular function, memory, and measured fluid intelligence. ${ }^{181}$ Congruent with the 'perception of action' hypothesis specialization, atypical subjects' right-hemisphere representations and associated cognitive processes may provide an advantage in complex matching tasks using perceptual recognition and integration of complex motor informational schemas. This memory for action specialization, rather than memory for declarative content as typified by the cognitive processes tapped by traditional IQ tests, could be explained by any number of plausible hypotheses.

There are other reasons as to why in the vast majority of crossed aphasia in dextral subjects might have separate praxis and language localization. By virtue of atypical subject connectivity to bilateral motor response mechanisms, it might be possible for such subjects to demonstrate superiority in complex verbal-motor perceptual matching tasks. For instance, the right hemisphere is known: (i) to elicit broader activation of semantic categories (ii) far greater sensitivity to distant prime-target pairs (iii) involvement in implicit rather than explicit retrieval (iv) usefulness for predictive inferences (v) for application of knowledge (vi) for lengthy latency of activation to primes (vii) for more diffuse and wider interconnectivity (viii) for better use of strategy (ix) for propensity for global aspects of language, and (x) for greater facility for ambiguous discourse. ${ }^{2}$ Further consideration of right hemisphere dominant atypical subjects and semantic networks; handedness; unusual praxic co-localization could suggest methods by which these unique subjects could be studied in the future.

\section{Praxis lateralization and handedness in anomalous subjects}

Aphasia after right hemisphere damage is greater in lefthanders than in right-handers and occurs with a frequency of approximately 30\%. ${ }^{182}$ As Alexander and Annett hypothesized: 183 "... The [rights shift theory] proposes to explain the typical pattern of cerebral asymmetry and normal variations as due to the presence and absence of a single gene ( $r s+)$. This gene confers some relative advantages on the left hemisphere which induces the left side to serve speech and also gives a relative advantage to the right hand. It does not determine handedness, but it shifts a chance distribution of handedness toward dextrality. In the absence of the gene (rs-genotype) lateralizations of language and of handedness, and presumably of all other lateralized functions, occur by chance (p. 216)." One assumption pertaining to this hypothesis is that in the population of such subjects, all possible configurations of cognitive modules should be found.

Most subjects then with right hemisphere dominance for language show left-hemisphere lateralization for praxis; an uncommon dissociation the reason for which appears unknown. ${ }^{174}$ It is possible that evolutionarily such a dislocation might have enabled accurate unimanual throwing whilst conversing with other group members. This hypothesis would suggest that language and visuospatial mechanisms would be parcellated into opposite hemispheres. Ninety-two percent of crossed aphasics showed evidence of this pattern. ${ }^{174}$ One survey of 73 left-handers with aphasia found that not one subject with right brain damage had limb apraxia. ${ }^{184}$ Other studies showed that $70 \%$ of left-handed subjects were left hemisphere dominant for language, 17\% were right hemisphere dominant for linguistic functions and that only $13 \%$ were bilaterally represented. ${ }^{185}$ Finally good praxis/language studies of this nature should contain measures of: (i) handedness and limb praxis (ii) language localization and buccofacial praxis and (iii) visuospatial disorders and neglect. ${ }^{186}$

Finally, when comparing measures of handedness in women and men, the right-shift was found to be of greater magnitude in women. ${ }^{187}$ However, it is noteworthy that, to date, comprehensive quantitative trait loci (QTL) studies of 
handedness have not been completed. Such studies have the potential to verify which gene(s) underlie handedness and hence linguistic/praxis dominance across the genders. ${ }^{187}$ This feminine difference is congruent with the hypothesis that when the RS gene is absent (rs -) in women, there is a higher incidence of random lateralization, thereby precipitating a heightened propensity towards atypical, anomalous, or mirror symmetric localization. Conceivably, this might occur through expression of a recessive trait, perhaps implying a predominant maternal influence in eventual phenotypic expression of atypicality and in strong right hemisphere lateralization. Combined QTL and fMRI would perhaps be able to determine if atypicality is indeed associated with unique co-localization of cognitive functions.

\section{"Resting state" functional lateralization in anomalous subjects}

Since the mid-1990's the right hemisphere has been hypothesized to be the seat of insight. ${ }^{10}$ A summary of these early cognitive neuropsychological studies is important because these provided the theoretical basis for most functional neuroimaging insight studies, and its sister cousin intuition, conducted in recent years. Four direct parallels are acknowledged between cognition in insight and the right hemisphere. These include: (1) reliance on nonverbal processes and neural networks; (2) avoidance of functional fixedness or perseveration; (3) access to either nondominant interpretations, larger semantic networks and/or topological similarity in concepts; and (4) perceptual restructuring..$^{10}$ Our focus is on insight because (i) it is the more complex and is directly associated with eminent achievement and (ii) it has been neuropsychologically examined over a longer time period. Although; the right hemisphere is unambiguously dominant in insight and to a lesser extent intuitive processes we must stress that such differences are relative rather than absolute. ${ }^{188}$ Both the left and right hemispheres are often, but not always, involved in both processes; however the right hemisphere has a dominant role.

Verbal overshadowing experiments confirmed the detrimental effects of verbalization on visual memory, visual image processing, and decision-making. ${ }^{189}$ Verbalization impaired subjects' performance on insight, but not on logical problems, ${ }^{190}$ suggesting that insight relies more on nonreportable processes. Moreover, protocol analysis found argumentative structures in logical problems and frequent pauses and lack of articulation associated with insight problems. ${ }^{107} \mathrm{In}$ a similar manner, "functional fixedness" describes a predisposition to use a tool or object to make a transitive action in a standard manner. In other words, the pre-potent action that an object affords is the dominant action, and not a nonstandard and novel action. However, subjects can learn to use an object in a nonstandard manner that is inconsistent with its more typical use, by overcoming the inherent functional fixedness ascribed to the object. Such cognitive biases persist despite insightful solutions that in hindsight, may appear simple, and may serve to block the participant's search for alternative solution strategies.

Various concept formation tasks are related to insight problems. Patients with right anterior damage made more perseverative errors than left anterior patients on the Wisconsin Card Sort test. ${ }^{191}$ Patients with right, but not left anterior temporal lesions, made more such errors. ${ }^{192}$ Patients with right hemisphere lesions made more perseverative errors; ${ }^{193}$ as did those with right temporal lobe epileptic foci. ${ }^{194}$ Novel and nonobvious alternative solutions to insight problems appear to involve both the right frontal and right anterior temporal structures, with access to nondominant meanings of critical words often essential. In insight often alternative interpretations are modeled by "spreading activation" theories of memory retrieval. ${ }^{195}$ Participants who were unable to generate the correct word in a mini-insight problem, exhibited subconscious activation profiles to such an extent that the incorrect guess was nevertheless still related to the correct cue. ${ }^{196}$ Finally, the right anterior locus of both perseveration and access to nondominant meanings, are consistent with fMRI studies showing activation during memory retrieval. ${ }^{197}$ In addition to allowing for access to multiple meanings, there is evidence that the right hemisphere encodes meaning at a much broader and expansive level. ${ }^{50}$ Left visual field presentations resulted in substantially more priming to distantly related words in a divided field paradigm than right visual field presentations. ${ }^{50}$ Similarly, the right hemisphere benefited more from interconceptual relations (eg, pear, plum) than from intraconceptual relations (eg, tree, roots). ${ }^{198}$ The left hemisphere referents were determined by logical/classificatory links, whereas right hemisphere referents were associated with situational links. Finally, in the left hemisphere there is rapid selection of one meaning and suppression of other candidate meanings, while activation spreads more diffusely and slowly within the distributed right hemispheric semantic network. ${ }^{199}$

Consonant with more diffuse activation within the right hemisphere, are findings that the left hemisphere contains more large pyramidal cells. ${ }^{200}$ These authors note that: “...even though both cell-column spacing and dendritic spread change in the same direction within the left hemi- 
sphere, within language-associated regions, they do not change proportionally. Therefore, individual pyramidal cells in each hemisphere contact a different number of adjacent cell columns. In the left hemisphere, this asymmetry results in a smaller number of interconnected columns than in the right, and it has been suggested that this might indicate a more elaborate and less redundant pattern of local processing architecture in the left hemisphere... (p. 431)." ${ }^{.201}$ The authors review ample contemporary neurobiological evidence which confirms that differing numbers of macrocolumnar systems, between the left and right hemispheres, allows for differently tuned systems, available to analyze information on a fine or coarse scale.

Perceptual restructuring is analogous to gestalt parallel recognition processes in which an object's identity is suddenly perceived. There is a direct neuropsychological equivalent to perceptual restructuring. Integrative agnosia is a relatively rare disorder that occurs when a patient has difficulty integrating disparate elements of a display into a coherent singular gestalt form. In this sense, integrative agnosia is the opposite of what occurs during perceptual restructuring. In one of the most comprehensive studies of such patients, it was found that 5 of 7 subjects demonstrated right hemisphere injury ( 3 of 7 right temporal); half were poor at figure-ground segregation; all were poor at discriminating overlapping figures; and most demonstrated deficits in amodal completion; perception of illusory contours; and in grouping processes. ${ }^{202}$ Perceptual restructuring in insight has been shown to be analogous to such perceptual pattern recognition processes. ${ }^{107}$ Scholastic Aptitude Test and 11 other potential correlates of successful insight problem solving were analyzed and only object contour drawings and the Embedded Figures Test (EFT) ${ }^{203}$ were highly significant predictors. ${ }^{202}$ Concurrent verbalization while solving the EFT impaired the ability to make such correct perceptual identifications. ${ }^{10}$

There are good reasons to suspect that the habitual processing mode of the right hemisphere is fundamentally different from that of the left. ${ }^{188}$ Considerable evidence points to right hemisphere superiority at: imagery, ${ }^{204}$ paradigmatic, ${ }^{205}$ simultaneous, ${ }^{206}$ global, ${ }^{207}$ coordinate, ${ }^{208}$ parallel,${ }^{209}$ and holistic ${ }^{11}$ processing. ${ }^{210}$ Baynes and Gazzaniga's discussion of the left hemisphere "interpreter" also highlights the situational interpretation superiority of the right hemisphere in split-brain patients. ${ }^{211}$ Whether similar mechanisms exist in the normal right hemisphere or for that matter in right hemisphere language dominant subjects is open to debate. The interpreter then seeks explanations for why events occur in a "make it up as we go along" manner; whereas the right hemisphere demonstrates an acute awareness for more disparate contextual factors, affective reactions as these relate to self." ${ }^{211}$

The difficulty then is determining just what the functions of this right hemisphere are compared to that of this logicogrammatical interpreter of the left hemisphere? Since most normal subjects do not have well-developed linguistic functions in the right hemisphere it is difficult to ascertain just what exactly the right hemisphere is doing compared with the left hemisphere interpreter. Indeed as Baynes and Gazzaniga note; philosopher of consciousness Daniel Dennett's practical comments on this issue by suggesting that researchers “...accurately assess the upper extent of [right hemisphere] cognitive function... (p. 1355)." ${ }^{211}$ Incidentally, hints into insight problems, presented to the right hemisphere, were solved better. ${ }^{10}$ The right hemisphere advantage interacted with a delay, such that the hint effect was substantially larger when individuals were working on the problem unsuccessfully for at least two minutes. ${ }^{10}$ Collectively, this suggests that right hemisphere processes are particularly apt to be involved in insight problem-solving, when individuals are stuck in set. The right hemisphere is involved in insight problem-solving, through avoidance of perseveration, access to nondominant meaning and longer-term "problem-finding".

Relatedly, positive mood has been found to alter preparatory activity within the dorsal anterior cingulate and right angular gyrus and thereby bias participants to engage in processing favorable to insight as opposed to analytical problem solving. ${ }^{212}$ Positive affect tips the balance towards global as opposed to local foci of attention ${ }^{213}$ and towards broader attention to either external visual space or internal conceptual space. ${ }^{214}$ Positive affect appears to heighten solvers' sensitivity to right hemispheric semantic processing, in conjunction with a frontal-mediated cognitive control network presumably involved in problem restructuring. Recent work on functional lateralization has emphasized the importance of whole hemisphere level coordinative functions of top-down frontal mediated brain networks ${ }^{215}$ purportedly involved in the development of right hemisphere language dominance. As an example, the anterior cingulate is involved in overcoming pre-potent responses when conflict is high and strategic processes are less engaged ${ }^{216}$ and the right angular gyrus is involved in spatial attention. ${ }^{217}$ This right hemispheric network appears to function in the de-selection of pre-potent responses, restructuring of the solution relevant activations and biasing responses towards global internal and/or external attentional loci.

Resting state EGG activity was compared between subjects classified as high insight or low insight using solution 
rates to anagrams. ${ }^{218,219}$ Few studies have tested the hypothesis that individuals differ on a factor such as lateralization of function, with the exception of spontaneous undirected thought. ${ }^{220}$ An EEG study found that alpha band activity distinguished between individuals with high verbal aptitude and those with high nonverbal aptitude. ${ }^{221}$ Verbalizers made greater use of the left parietal regions, whereas nonverbal strategizers made greater use of the right parietal lobe. Correlational analyses supported the position that insight self-reports actually reflected differential use of problem solving strategies, and there was greater bilateral occipital beta $(13-18 \mathrm{~Hz})$ indicative of more focused visual attention for low insight subjects. ${ }^{218,219}$ Hence individual differences in resting state brain activity directly influenced the neural computations associated with insight and this finding is consistent with a diffuse attention mode of highly creative individuals. ${ }^{222}$ Greater low alpha at right dorsal-frontal, beta and gamma at right inferior frontal, as well as greater gamma at right parietal was consistent with right functional lateralization in high insight subjects. ${ }^{218,219}$

Discourse processes, that place a premium on computing distant semantic relations, are particularly apt to result in increases in neural activity within the right temporal lobe, ${ }^{223}$ and especially in right anterior-superior temporal lobe. ${ }^{224}$ Therefore, the nonverbal nature of intuition and insight suggests a "building block" relationship between the constructs. Intuitive and insightful processes could be used preferentially and habitually, as a function of the fact that they are the preferred cognitive modes for the individual. However, on closer examination, the preferred cognitive mode scenario usually arises as an indirect developmental consequence of specific ability endowment, such as verbal, nonverbal, or spatial second-order factors of intelligence. ${ }^{225}$ Alternatively, preferred cognitive mode could be determined by functional lateralization indices. ${ }^{177,226-228}$

Our focus now turns to this developmental hypothesis of the origins of right hemisphere language. Performance on covert visual orientating which is a measure of attentional asymmetry, ${ }^{229}$ and the Raven standard progressive matrices ${ }^{230}$ was correlated. ${ }^{231}$ High asymmetry was negatively correlated with the sine que non measure of fluid intelligence, as measured by the Raven. Similarly an ear advantage in either direction in sinistrals, was associated with lower Object Assembly scores on the WAIS. ${ }^{232}$ And more specifically, the achievement-ability domain scores on the Medical College Admission Test were correlated with gender and handedness. ${ }^{233}$ Right hemisphere dominance was associated with intellectual giftedness in verbal reasoning and these right-handed individuals scored higher on writing indices. Superior intelligence was associated with stronger recruitment of the bilateral posterior parietal cortex. ${ }^{234}$ High IQ has also been associated with bilateral parietal integrity, ${ }^{235}$ which re-directs us to the question of the functional relationship between bilateral frontoparietal networks, and the posited ventral right semantic stream associated with intuition and insight.

\section{IQ achievement discrepancy and anomalous subjects}

In order to find subjects with strong asymmetry in the direction of the right hemisphere, with little or no bilateralism of functions, large samples of several hundred subjects are required to be sifted through. This search requires that a preliminary method of determining functional lateralization that is cheap and efficient be available. FMRI and PET are too expensive for preliminary first round searches unless the data is associated with a few of the world's largest functional neuroimaging centers. Even at these centers finding atypical subjects may be difficult since the data may have been collected over decades making it difficult to track down subjects. Also many imaging protocols have not used full brain scans. Functional transcranial doppler sonography or fTCD has been validated in conjunction with intracarotid amobarbitol injection, or the WADA procedure, ${ }^{226}$ as well as $\mathrm{fMRI}^{236}$ for determining the direction and degree of functional lateralization of cognitive functions. Functional TCD constitutes a noninvasive, cheap, quick, and reliable method of determining lateralization in large samples.

Archival fMRI data collected at the Massachusetts General Hospital Functional Neuroimaging Center identified several strongly right hemisphere dominant subjects. ${ }^{7}$ These investigators conducted neuropsychological examination of three subjects all of whom happened to be female. A difference in favor of performance IQ versus verbal IQ was found in all three subjects without any history of confounding neurological illness. The investigators noted that in the past the vast majority of patients studied with right hemisphere language had a pre-existing neuropathology. These authors advocated further study in the area, particularly in right hemisphere language dominant women, since most studies have examined ostensibly neurologically pathological individuals. ${ }^{7}$ In the Chee and Caplan study, it was noted that both verbal and performance scales were in the normal range for all three healthy subjects. ${ }^{7}$ The authors also observed that a complete mirror reversal of cognitive functions occurs in at least a subset of subjects; what this means in terms of adaptive functioning per se is not yet 
completely understood. Interestingly, the three female subjects studied were professionals or were employed in skilled occupations, requiring high levels of education and advanced post-secondary training. A complete reversal of language and face encoding was found in subject 1 , and partial reversal of these functions in subjects 2 and 3 . The dislocation of language and face encoding in one subject, and the co-location of face encoding and language in two subjects, provides ambiguous data in reference to the validity of the crowding hypothesis. Z-scores were calculated and pooled across for subjects 2 and 3 . The differences in subject 3 in terms of VIQ and PIQ could be interpreted as some support for the crowding hypothesis. ${ }^{7,37}$ In fact the z-scores for subjects 2 and 3, with incomplete reversal of cognitive functioning, show that when IQ is constant, the only definitive conclusion is that the individuals demonstrated superior Block Design scores, which in contrary to the 'crowding hypothesis'. All individuals were presumed of superior intellectual ability, given advanced postsecondary achievement indicators (Table 1).

Table 1 shows that in partial mirror reversal of lateralization, there was better performance on the Block Design subtest of the WAIS-R when compared with that patient with complete mirror reversal. Block Design is generally considered the best measure of visuospatial perceptual organization in the Wechsler scales, ${ }^{238}$ and lesions of the parietal lobes are most prone to result in reliable decrements in performance on this scale. ${ }^{235}$ Since performance was greater in subjects with incomplete mirror reversal, in spite of approximately equivalent performance on the VIQ,

Table I Comparison of complete versus partial reversal of cognitive functions in atypical language lateralization subjects. Copyright (C) 200I. Adapted and reprinted with permission of Lippincott, Williams \& Wilkins, Ltd. from Chee MWL, Caplan D. Face encoding and psychometric testing in healthy dextral with right hemisphere language. Neurology. 59(I2):1928-1934

\begin{tabular}{|c|c|c|c|c|c|}
\hline $\begin{array}{l}\text { Subject } \\
\text { variables }\end{array}$ & Subject I & Subject 2 & Subject 3 & $\begin{array}{l}2 \text { and } 3 \\
\text { average difference }\end{array}$ & $\begin{array}{l}\text { Z-score } \\
\text { 'versus } \mathbf{x}^{2+3}\end{array}$ \\
\hline Age & 23 & 25 & 24 & 25 & - \\
\hline Gender & Female & Female & Female & Female & - \\
\hline Educ & College & High school & College & College & - \\
\hline Hand & $+23 / 24(\mathrm{R})$ & $+9 / 24(\mathrm{R})$ & $+19 / 24(\mathrm{R})$ & $+14 / 24(R)$ & 0.38 \\
\hline Face Encod & $+0.44 / 1.00$ & $+0.08 / 1.00$ & $+0.05 / 1.00$ & $+0.07 / 1.00$ & 0.44 \\
\hline Word Encod & $-0.96 / 1.00$ & $-1.00 / 1.00$ & $-0.70 / 1.00$ & $-0.85 / 1.00$ & 0.11 \\
\hline Mirror Rev & Complete & Partial & Partial & Partial & - \\
\hline WAIS FSIQ & $z=+0.46$ & $z=-0.20$ & $z=+1.07$ & $z=+0.44$ & - \\
\hline WAIS VIQ & $z=+0.20$ & $z=-0.67$ & $z=+0.27$ & $z=-0.20$ & - \\
\hline WAIS PIQ & $z=+0.93$ & $z=+0.53$ & $z=+1.93$ & $z=+I .23$ & - \\
\hline WAIS BD & $z=0.00$ & $z=+1.67$ & $z=+3.00$ & $z=+2.34$ & $2.34 P<0.01$ \\
\hline WAIS DS & $z=+1.67$ & $z=+1.33$ & $z=+2.00$ & $z=+1.67$ & - \\
\hline Word Mem & $z=+I .30$ & $z=+1.30$ & $z=+1.30$ & $z=+I .30$ & - \\
\hline Face Mem & $z=+0.30$ & $z=0.00$ & $z=+0.50$ & $z=0.25$ & - \\
\hline Imm Rec Vs & $z=+0.70$ & $z=+I .70$ & $z=+1.70$ & $z=+I .70$ & - \\
\hline Del Rec Vs & $z=+1.10$ & $z=+2.50$ & $z=+2.50$ & $z=+2.50$ & $1.40 P<0.10$ \\
\hline Imm Rec Ve & $z=+0.60$ & $z=0.00$ & $z=+0.30$ & $z=+0.15$ & - \\
\hline Del RecVe & $z=+0.20$ & $z=+0.20$ & $z=+0.70$ & $z=+0.45$ & - \\
\hline Vis Mem I & $z=+1.33$ & $z=+0.87$ & $z=+0.07$ & $z=+0.47$ & - \\
\hline Ver Mem I & $z=+0.60$ & $z=-0.13$ & $z=+0.13$ & $z=0.00$ & - \\
\hline ROFR & $z=+1.86$ & $z=+0.64$ & $z=+0.79$ & $z=+0.72$ & - \\
\hline
\end{tabular}

Notes: Bold type indicates potential statistical differences. On the frontal face encoding and the frontal word encoding a positive value denoted left hemispheric dominance and a negative value denotes right hemispheric dominance.

Abbreviations: Educ, education; Hand, handedness; Face Encod, frontal face encoding; Word Encod, frontal word encoding; Mirror Rev, mirror reversal; WAIS, Wechsler Adult Intelligence Scale-Revised; VIQ, verbal IQ; PIQ, performance IQ; BD, block design; DS, digit symbol; Word Mem, word memory; WRMT, Warrington Recognition Memory Test; Face Mem, face memory; Imm RecVs, immediate recall (visual); Del RecVs, delayed recall (visual); Imm Rec Ve, immediate recall (verbal); Del RecVe, delayed recall (verbal); Vis Mem I, visual memory index; Ver Mem I, verbal memory index; RORF, Rey-Osterrieth Figure Recall. 
PIQ, and FSIQ, the findings are congruent with the assertion that crowding is not by any means the norm among these subjects. Usually right lateralization is found for spatial attention, ${ }^{239}$ and sometimes neglect is found after left hemisphere lesions in select subjects. ${ }^{240}$ No differences in verbal fluency or line bisection performance were found between the left and right hemispheres of atypically localized subjects when compared with those of normal lateralization pattern subjects. ${ }^{8}$ Unfortunately this sample recruited preferentially nonright-handers, unlike the Chee and Caplan study, ${ }^{7}$ and this added additional complexity to the interpretation of their results. ${ }^{8}$ However, a secondary analysis assists in clarification of the mechanisms operating within these data. Table 2 shows that among right-handers, there was a greater number of males with complete mirror reversal and those members exhibited a greater degree of mirror reversal. Whether this pattern holds for larger samples would depend upon an analysis of more right hemisphere dominant dextrals (Table 2).

Further analysis of the subset $(n=4 / 10)$ with incomplete mirror reversal, may help clarify this pattern of results found among the largest subset of subjects with demonstrated unambiguous right hemisphere language. To this end then, the short form of the German IQ test (Leistungsprufensystem [LPS]) for adults aged less than 50 years, was used to test general intellectual functioning. ${ }^{241}$ The detailed study revealed that two medical students, a teacher, and a business consultant scored within the first standard deviation unit on an equivalent WAIS-R FSIQ measure which is surprisingly low. One female medical student who was right-handed, scored highest on LPS reasoning as well as on the degree of right hemispheric language lateralization. The superior achievement indices

Table 2 Handedness and frequencies of demographic, lateralization and performance parameters ${ }^{8}$

\begin{tabular}{lll}
\hline Demographics and tests & Left-handers & Right-handers \\
\hline Age & 26 & 27 \\
Number & $(\mathrm{n}=7) 70 \%$ & $(\mathrm{n}=3) 30 \%$ \\
Gender & $43 \%$ Male & $\mathbf{6 7 \%}$ Male \\
Handedness & -72 & +74 \\
Language lateralization & -3.49 & -3.37 \\
Attentional lateralization & +0.83 & +1.07 \\
Complete mirror reversal & $57 \%$ & $\mathbf{6 7 \%}$ \\
Verbal fluency & $\mathrm{z}=+1.82$ & $\mathrm{z}=+2.38$ \\
Line bisection & $\mathrm{z}=+0.02$ & $\mathrm{z}=+0.15$ \\
\hline
\end{tabular}

Notes: Bold type indicates potential statistical differences. Positive values for language and attentional lateralization denote left hemispheric dominance whereas negative values denote right hemispheric dominance. of physician, business person, and teacher suggest that these unique subjects possess a type of cognitive advantage in a specific ability as opposed to that of IQ. ${ }^{242}$ Collectively, these findings contradict the crowding hypothesis but do point to the puzzlingly low FSIQ indices in some subjects. Finally, in the earlier study by Knecht and colleagues, upon which this study was based, in a atypically localized sample of subjects $(n=21$ of 326$)$, no correlation was found between FSIQ and language lateralization. ${ }^{227}$ Across the full-range of laterality indices, one striking finding was that a subject with the most extreme rightward lateralization index, also scored highest with an IQ of greater than 150. The latter finding suggests that aptitude-achievement discrepancies are not necessarily the norm, in such atypical subjects, but rather could involve more complex factors (eg, co-association or compensatory development of alternative specific abilities or capacities). However, it is becoming increasingly clear that atypical language dominance is not by any means intrinsically pathological as (i) classical neurolinguistic theory (ii) conventional clinical neuropsychological wisdom or (iii) older empirical studies would suggest (Table 3).

\section{Communicative superiority and functional lateralization?}

These early descriptive and qualitative studies constitute very small samples however these are nonetheless quite provocative. The functional significance of these atypical and anomalous subjects' patterns of laterality is still largely unclear. Knecht and colleagues conducted an elegant functional MRI study of seven left hemisphere and seven right hemisphere language dominant subjects. ${ }^{228} \mathrm{~A}$ uniform and reliable finding was that there was no evidence of increased bilaterality of linguistic functions in right hemisphere-dominant (RHD) subjects. More telling was that no more variability was found in the loci of activation in RHD subjects. The last conclusion is significant since it runs contrary to several developmental psychopathology accounts of the origins of extreme RHD. ${ }^{243}$ In other words, the two patterns of spatial topography of hemispheric activation, were completely mirror symmetric. Similarly, there was no evidence of the compensatory bilateral activation that might be expected on the basis of studies of early injury to cortical or mixed cortical/subcortical regions.

When flipping the activation patterns for left hemispheredominant (LHD) and RHD and using comparisons, no differences in either the first or second fMRI activation profiles were found, which are results that supported the 
Table 3 Handedness and frequencies of demographics, lateralization and performance parameters for 4 subjects with incomplete mirror reversal. Copyright (C) 200I. Adapted and reprinted with permission of Lippincott,Williams \&Wilkins, Ltd. From Floel A, Knecht S, Lohmann H, et al. Language and spatial attention can lateralize to the same hemisphere in healthy humans. Neurology. 2001;57:1018-1024

\begin{tabular}{|c|c|c|c|c|c|}
\hline Demographics & Subject I & Subject 2 & Subject 3 & Subject 4 & Average and tests \\
\hline FSIQ & $z=+0.60$ & $z=+0.40$ & $z=+0.50$ & $z=+0.60$ & $z=+0.53$ \\
\hline Occupation & Medical student & Business consultant & Teacher & Medical Student & \\
\hline Age & 25 & 32 & 28 & 23 & 27 \\
\hline Gender & M & $\mathrm{F}$ & $\mathrm{F}$ & $\mathrm{F}$ & $75 \% \mathrm{~F}$ \\
\hline Handedness & $-30(L)$ & $-100(\mathrm{~L})$ & $-23(\mathrm{~L})$ & $+79(R)$ & $75 \%$ LH \\
\hline Lang. Lat. & $-1.73(\mathrm{R})$ & $-3.06(R)$ & $-3.36(R)$ & $-4.13(R)$ & $-3.07(\mathrm{R})$ \\
\hline Att. Lat. & $-I .7 I(R)$ & $-2.78(\mathrm{R})$ & $-3.20(\mathrm{R})$ & $-2.86(R)$ & $-2.64(R)$ \\
\hline Incomplete Mirror R. & $\sqrt{ }$ & $\sqrt{ }$ & $\sqrt{ }$ & $\sqrt{ }$ & All Subjects \\
\hline Verbal Fluency & $z=+2.29$ & $z=+1.26$ & $z=+1.92$ & $z=+2.48$ & $z=+2.01$ \\
\hline Line Bisection & $z=+0.02$ & $z=-0.54$ & $z=+0.02$ & $z=-1.96$ & $z=-0.67$ \\
\hline LPS V.K. & $z=0.00$ & $z=0.00$ & $z=+0.50$ & $z=0.00$ & $z=+0.13$ \\
\hline LPS Reasoning & $z=+1.00$ & $z=0.00$ & $z=+1.00$ & $z=+1.30$ & $z=+0.83$ \\
\hline LPSW.F. & $z=+1.30$ & $z=+0.50$ & $z=+0.50$ & $z=+1.30$ & $z=+0.90$ \\
\hline LPS V.P. & $z=+0.50$ & $z=+0.50$ & $z=0.00$ & $z=+0.50$ & $z=+0.38$ \\
\hline LPS Figure-Ground & $z=+0.50$ & $z=+1.00$ & $z=+0.50$ & $z=+1.30$ & $z=+0.83$ \\
\hline LPS Flex. of Closure & $z=+0.50$ & $z=+0.50$ & $z=+0.50$ & $z=+0.00$ & $z=+0.38$ \\
\hline WAIS-R D.S. & $z=-0.70$ & $z=0.00$ & $z=-1.00$ & $z=+0.20$ & $z=-0.38$ \\
\hline Corsi Block Span & $z=+0.50$ & $z=+0.50$ & $z=-0.70$ & $z=+0.20$ & $z=+0.13$ \\
\hline Trail-Making A/B & $z=+1.30$ & $z=+0.70$ & $z=-0.20$ & $z=+1.30$ & $z=+0.78$ \\
\hline Speed/Accuracy & $z=+0.70$ & $z=+I .40$ & $z=+0.50$ & $z=+1.60$ & $z=+1.05$ \\
\hline Finger Tapping (RH) & - & $+2.78 \mathrm{LH}$ & $+3.20 \mathrm{LH}$ & $+2.86 \mathrm{LH}$ & $+2.2 \mathrm{I}(\mathrm{LH})$ \\
\hline EEG Scan & Normal & Normal & Normal & Normal & All normal \\
\hline
\end{tabular}

Note: Bold type indicates potential statistical differences.

Abbreviations: FSIQ, Leistungsprufsystem (LPS) Full-Scale IQ; Lang. Lat., Language Lateralization; Att. Lat., Attentional Lateralization; Incomplete Mirror R., Incomplete Mirror Reversal; LPS V.K., LPS Verbal Knowledge; LPS W.F., LPS Word Fluency; LPS V.P., LPS Visual Perception; LPS Figure-Ground, LPS Figure-Background Perception; LPS Flex. of Closure, LPS Flexibility of Closure; WAIS-R D.S.,Wechsler Adult Intelligence Scale - Revised Digit Span Subtest; Corsi Block Span, Corsi Block Tapping Span; Trail-Making A/B, Trail-Making Test Part A (Cognitive Speed) and TMT Part B (Mental Flexibility) Combined Score; Speed-Accuracy, Concentration: Speed/ Accuracy d2 test; Finger Tapping $(\mathrm{RH})$, Finger Tapping Praxis Localization using the Right Hand.

mirror hypothesis, at least for healthy normal RHD subjects. In fact, for strongly RHD subjects there was greater right lateralization of linguistic processes than was found for leftward lateralization in LHD subjects! Finally, the review by Woods and colleagues suggests that complete shifts of cerebral lateralization only occur after early and extensive cortical and noncortical structural or functional lesions. ${ }^{243}$ Such a pathological hypothesis and hence pattern would be expected to be associated with spatially disorganized mapping. However, the subjects in this study exhibited normal structural and functional MRI scans, as well as normal mirror symmetry. Since the 14 subjects were recruited from a consecutive normal population sample of 326 , the findings were interpreted by the European Munster Functional Imaging Study group as strong evidence against the early pathology account of RHD.
Developmental neuropsychological studies are of a similar opinion that anomalous patterns are determined genetically and may arise in the uterine environment. ${ }^{244}$ Recent studies examined, not only the typicality of linguistic lateralization in right hemisphere dominant individuals, but also the theoretically important issue of the co-dominance patterns of association in these rare subjects. Floel and colleagues' study was among the first to examine the spatial topography, hemisphericity, and intrahemispheric activation pattern of spatial attention in RHD. ${ }^{5}$ The left intrahemispheric activation pattern for spatial attention was found homotopic, with regular spatial topography of activation for right hemisphere dominant spatial attention processes! Secondly, the authors discovered that in $\mathrm{RH}$ co-dominance subjects, the pattern of spatial attentional activation did not differ from subjects with right hemisphere dominance for 
only spatial attention or language! Finally, it was observed that RH co-dominance subjects in the study had no appreciable impairment in cognitive functioning. Consistent with the spatial topography of intrahemispheric RH language, the results suggest that it is the "intrahemispheric pattern", rather than the hemispheric side per se, that appears to be a hardwired pattern of brain organization. These convergent finding contradicts the leftward language acquisition device doctrine. $^{4}$

\section{Practical applications of intuition and insight}

Perhaps there are no more requirements for practical application of intuition and insight than in clinical psychological interviewing and assessment. An interesting example of how intuition and insight can be integrated is in the use of projective drawings to examine personality constructs. These sensitive projective tests can also be used to assess more distantly related psychodynamic defense mechanisms in patients and clients. Projective drawings such as the House-Tree-Person have been used in psychodiagnostics for well over 60 years ${ }^{245-247}$ and can be used for evaluation of psychopathology as well as evaluation of positive aspects of adaptation. Extensive normative and empirical studies are available for all ages and types of diagnosis. Patients are asked to draw a house, a tree, and a person in succession in an open-ended format and then the drawings are rigorously analyzed and scored for various specific test signs and "constellations of signs" usually taken to be indicative of a syndrome. This requires keen observation of the drawings in the context of the referral question, clinical history, other test results and the clinical interview. This is the epitomy of practical intuition of what fits and what does not fit into a clinical picture using a type of "semantic gestalt."

The three drawings depicted below were drawn by three separate adults. Usually patients will draw the full set of three drawings which each can be scored individually and then similarities in clinical themes across the three drawings can then be used to extrapolate "a configuration of signs". In the case of drawing A the house is unrecognizable and consists of what looks like a vague floor-plan with logical errors of missing walls and many mis-proportioned segments. These signs are symptomatic of organic brain damage and indeed drawing A was drawn by an adult with severe brain damage. Drawing B depicts two trees drawn in response to the request to draw $a$ tree. The shapes of the trees are decidedly masculine, depicted in a barren environment. The bimodal themes of hills and trees suggest dependency needs in addition to the over-compliance. Drawing $\mathrm{C}$ is a depiction of an individual with an extremely distorted body image, with the overall gestalt lines surrounding the face missing and with the macabre instance of intestines spilling out of the figure's abdomen. This drawing was done by a patient in the throes of an acute schizophrenic episode (Figure 8).

However we must emphasize that in recent years projective drawings have also been used to study factors associated with resiliency, normal adaptation, and positive features of personalities. One particularly excellent way to teach intuition

\section{A: House}

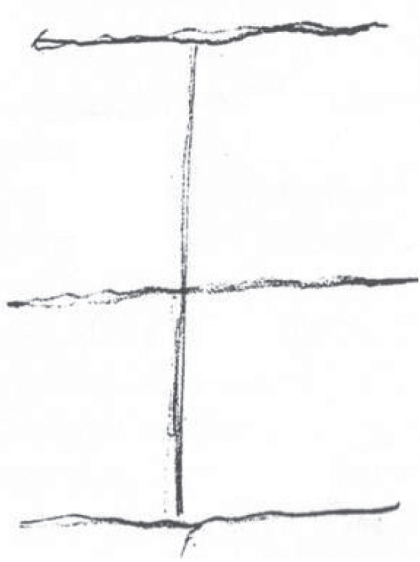

B: Tree

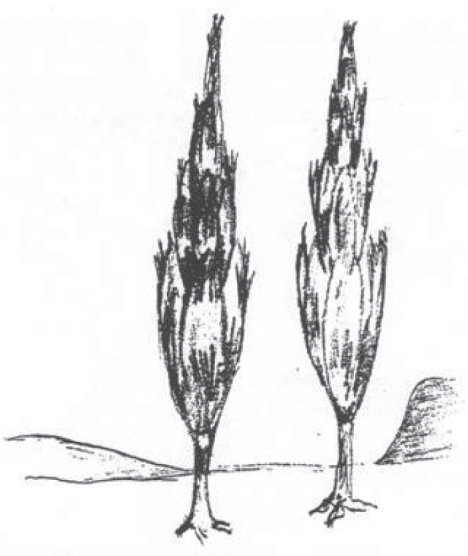

C: Person

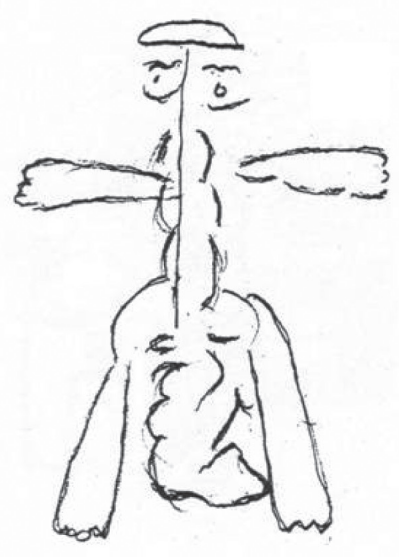

Figure 8 Projective drawings and personality inventory assessment as concordant examples of the relationship between intuition and insight in clinical psychology. Traditionally these projective tests have been used to characterize pathology but increasingly they are also being used to study normal developmental processes and healthy adaptation as in the positive psychology movement. Drawings are particularly apt to tap right hemispheric processes and notions of self, ${ }^{238}$ psychodynamic processes, ${ }^{245-247}$ and as hypothesized in this article links between conscious and unconscious mind. Copyright (C) 1997. From EH Hammer,Advances in Projective Drawing Interpretation, I997. Courtesy of Charles C.Thomas Publisher, Ltd., Springfield, Illinois. Drawing A is adapted from Figure 2-26 on page 34. Drawing B is adapted from Figure 5-7 on page 96 . Drawing C is adapted from Figure $2-16$ on page 25. 
to clinical psychology residents in the art of projectives is to ask the clinician to first get the patient to do the three sets of drawings. Then the individual drawings are scored for the separate House, Tree, and Person criteria. A thematic analysis of trends appearing across the three sets of drawings can then be extracted with usually a summary three line statement about personality structure and any salient defences latent in the drawings. This entire process in rich in projective intuitive interpretation. Note that during the course of the interview and subsequent formal testing the patient is usually asked to complete a standard personality inventory after the drawings: an objective personality test. Once the drawing summary is complete the clinician scores the personality inventory and looks at the degree of concordance between the subjective drawing summary and the objective personality profile. Correlation of drawings with standardized personality tests has been used as a clinical skill development technique since the 1960s. ${ }^{275}$ This "secondary insight-based process" is helpful in developing a diagnosis and case conceptualization for the client or patient in the context of referral question, clinical history, interview and the results of other standardized testing. Patient A would be expected to have a generally "flat profile" on the personality scales due to blunting of cognitive functions and general cognitive decline. Patient B would show elevations on measures indicative of dependency and Patient $\mathrm{C}$ would be expected to show elevations on somatization and thought disorder scales. Hence the entire process consists of two stages. Stage 1 involves a detailed analysis of the drawings in the context of objective scoring criteria and clinical intuition. Stage 2 involves an insight process that is more reflective in that the clinician takes all of the clinical information about the patient into consideration in formulating a tentative diagnosis, case conceptualization and treatment plan.

\section{Discussion}

In the last five years significant theoretical and empirical developments have advanced the understanding of behavioral, cognitive, and neuropsychological correlates and precursors of intuition and insight. Intuition is the ability to understand something immediately without need for conscious thought; whereas, insight is the capacity to gain an accurate and deep intuitive understanding of something. In popular culture, a link between the concepts has been alluded to. Now, however there are emerging case studies and cognitive and neural research results that add weight to the notion that the two concepts can be considered more sister cousins than orthogonal constructs. There are reliable individual differences found in insight and these differences have been associated with perception of relations and fluency; ${ }^{248}$ fluid intelligence, ${ }^{249}$ and spatial working memory. ${ }^{250}$ Similarly, individuals have been found to vary in intuitive acumen. Differences in nonverbal decoding appear central to most definitions of intuition and they have been recorded across a wide variety of study methodologies. ${ }^{114}$ Arguably, intuition often results in decisions that are as good or better than those arising from conscious deliberation, perhaps due in part to its structural properties..$^{30,190}$ Intuition has been found central to cognitive processes such as heuristic-based decision-making, ${ }^{251}$ creativity, ${ }^{17}$ and learning. ${ }^{25}$ The concept intuition has been correlated with successful and gifted performance in the practice of medicine and has been particularly associated with superior clinical decision-making. ${ }^{252}$ Intuition has been a utilized as a construct to explain success in many professions, ${ }^{253}$ including specific fields such as nursing ${ }^{254}$ and business management. ${ }^{255}$ Similarly, using the great minds approach, superior insight has been demonstrated to be pivotal to eminent achievement in many domains. ${ }^{36}$

To reiterate, intuition is automatic, nonconscious, affectively laden, absolute and judgmental, instant, implicit, associated with motor response, and the operation of right hemisphere nonverbal decoding mechanisms. In contrast, insight is effortful, conscious, affectively laden only at the moment of restructuring; nonabsolute and nonjudgmental, potentially occurring over longer time spans, declarative, associated with propositional reasoning, and like intuition with the operation of neural substrates in the right hemisphere. Whereas, intuition is associated with the control of expertise and automatized knowledge within basal ganglia and orbitofrontal networks, insight is associated with cognitive functions in knowledge-lean domains, restructuring within the right temporal lobe, and acting in conjunction with frontoparietal networks. It is interesting in this context that the first quantitative morphometric studies of right-hemisphere language dominant subjects only show greater grey matter volume in the right hippocampus. ${ }^{256}$ This is consistent with an emerging view that laterality of language localization is a function of maturation of the "what" ventral stream.

While intuition is associated with a single iterative stage, insight is characterized by the four progressive stages of preparation, incubation, insight and elaboration. Functionally and neurally, intuition is closely associated with the development of expertise, heuristics and affective gists during the preparation and incubation stages. The caudate and the orbitofrontal cortex are likely the means through which the two functional neuroanatomical systems 
interface and computationally interact. Moreover, it appears that intuition usurps declarative knowledge systems once implicit understandings become routinized and automatic via the striatum. Therefore, most perceptual restructuring that occurs during insights" "eureka" or "aha" moments are a function of subconscious transformation of informationally rich chunks or models. These chunks or intuitive schemas or models have a high throughput band-width which allows for coding vast amounts of information that may be easily modified in the future.

There is anecdotal, empirical, and neurophysiological evidence demonstrating a female advantage on intuitive type tasks involving nonverbal decoding. Also, self-knowledge systems and emotional appraisal are dependent upon effective interaction between basal ganglia and limbic neural networks, thereby demonstrating that self-perception is not completely reliant on declarative memory. Similarly, neuropragmatic tasks like determination of the order of events in social episodes are reliant on right orbitofrontal systems. Other pragmatic aspects of language such as mentalizing or theory of mind (right temporoparietal); ${ }^{257,258}$ inhibition of one's own experience during the consideration of another's state of mind (right ventrolateral prefrontal cortex); ${ }^{259}$ selfrecognition (right lateral prefrontal cortex); ${ }^{260}$ metaphoric figurative language; ${ }^{261}$ and indirect requests ${ }^{10}$ are similarly dependent on both posterior and anterior right hemispheric networks. Thus, intuition is, in a real sense, primary to insight in terms of the functional interaction of the two highly complex interdependent novel problem solving systems.

Since the right hemisphere is superior at imagery, ${ }^{204}$ global, ${ }^{207}$ coordinate, ${ }^{208}$ and situational as opposed to interpretive functions $;{ }^{211}$ intuition and insight in right hemisphere dominant subjects could give rise to "emergent properties." Utilizing functional transcranial doppler, a European research consortium tested hundreds of subjects for linguistic and spatial localization across the hemispheres. ${ }^{228}$ Several dozen female and male subjects, exhibiting extreme levels of right hemisphere language localization were discovered, and interestingly, the magnitude of rightward lateralization was greater than that of leftward lateralization. There was little concordance with the crowding hypothesis, which would have predicted that all subjects have co-extensive spatial and constructional praxis skills in the right linguistic hemisphere. Moreover, subsequent case studies of a cohort of the individuals, many of whom were female, confirmed superior achievement on measures of occupational attainment. This superior achievement was in the context of having recorded mediocre scores on conventionally measured IQ test.
The results show gender differences in specific functions as well as perhaps application of gender-specific psychometric testing protocols.

Finally Floel and colleagues' study of two healthy normal female subjects showed language and spatial functions lateralizing within the right hemisphere using $\mathrm{fMRI}^{5}$ with no adverse effects on cognitive functioning confirming the co-extensivity hypothesis. ${ }^{12,13}$ Similarly a 29 -year-old right-handed male student demonstrated total lateralization reversal as measured by abstract/concrete word decisions with visually and auditory presented words. ${ }^{6}$ The same pattern was found with a visual word generation task and notably cortical and cerebellar foci were mirror symmetric. Since this male subject had no history of brain damage the authors hypothesized that this pattern of brain organization was present from an early age and likely determined genetically. Concordant with this hypothesis, complementary frontocerebellar crossed functions in both left and right language dominant subjects, implies early maturing subcortical influences on the eventual manifestation of hemisphericity for linguistic and spatial processes. ${ }^{6}$ Hence, the documentation of co-extensivity of language and spatial processes, as well as unique co-configurations of modules in an unexpected hemisphere, without the necessity of crowding or attenuation of general cognitive functioning, does suggest that emergent cognitive functions is a possibility. That is language and spatial functions could interact in a single hemisphere in real-time with broad affects on the qualitative aspects of cognition that would not be present in normally brain organized subjects.

It is hypothesized that the achievement-ability discrepancies are congruent with speculation that the "functional capacity" of the subjects often exceeds their measured intelligence level. Incidentally, Colom and colleagues found that a right hemispheric network comprising right superior frontal gyrus and right inferior parietal lobule gray matter volume explained most of the variation on measures of working memory capacity in a large sample of individuals within the normal range of intelligence. ${ }^{262}$ Arguably, such individuals perform exceptionally well in intellectually demanding environments because, in addition to the regular cadre of cognitive abilities associated with typically localized subjects, the anomalous individuals possess emergent cognitive specializations that normal individuals either do not possess, or do not possess in abundance. The 'emergent properties' are hypothesized to be intuition, insight and social competence (and various permutations of these abilities in combination with semantic processing and motor responding), or perhaps "prefrontal-cerebellar inverse modeling mechanisms.",263 
A prefrontal-cerebellar inverse modeling mechanism could provide for the highly accurate mapping of semantic markers and related action concepts to visual gestures by recursive and iterative feedback occurring over ontogeny. ${ }^{263}$ It is hypothesized that this would amount to a much more articulate description of categories of human social interaction variables at a high degree of abstraction. ${ }^{264}$ As an example universals in sound-shape cross-domain mapping regardless of language, alphabet, or culture suggests that the brain may be similarly involved in extracting a common denominator between vision and movement ${ }^{265}$ and could be analogous to a semantic conceptual synaesthesia. ${ }^{266}$ A hardwired "crosslinguistic fluency" would aid in dispersal evolutionarily through cooperative behavior and might explain high general IQ ${ }^{249}$ and spatial IQ ${ }^{250}$ loadings. This would be akin to a tower of Babel effect. Unusual co-localization of language, emotion, and abstract semantics in the right hemisphere, while object-orientated actions and visuospatial functions reside in the left hemisphere could result in the developmental acquisition of emergent properties. In particular, the sequestration of praxis and concrete concepts to the left hemisphere could function as a superior signal-to-noise mapping mechanism, in concert with the right hemisphere's abstract linguistic mapping function. ${ }^{267}$ With this interpretation, the neuropsychological constructs of intuition and insight would be seen at the core of any popularized notion of 'emotional intelligence'.

The speculation that unique co-localization of cognitive modules, within a hemisphere, could developmentally confer a higher-order sociocognitive function specialization has been considered. In particular, the progression in theory of mind development and its hypothesized core role in virtually all aspects of the elaboration of normal higher-order sociocognitive functioning, ${ }^{268}$ as well as its dependence generally on advances in frontal-mediated inhibitory control, ${ }^{269}$ is telling. Such posteriorly located core theory of mind and biological motion detection networks within the right hemisphere must develop coherence while under the influence of prefrontal long-range connections. ${ }^{270}$ To test such a supposition however would require a systematic identification of subjects and a comprehensive neuropsychological evaluation longitudinally. Additionally, structural and functional neuroimaging would be required, as well as a cognitive neuropsychological analysis of each individual and the group as a whole. This approach would benefit from effective and functional connectivity studies to assist in determining if there were preferential cognitive processing modes associated with intuition and insight.
In summary intuition and insight, in close agreement with the connotation used by the general public and layman, are real psychological constructs at the upper end of complexity in the social cognition scale. Actual human performance is likely to consist of lengthy periods of didactic novice-expert learning of a skill which then becomes routinized and automatic, and is associated with intuition through a domain of knowledge. Similarly, insight is periodically associated with major conceptual transitions within a specific domain of knowledge, and it is punctuated by a regular and normally laborious series of propositional reasoning steps, followed by elaboration. Finally, automatic and subconscious intuitive knowledge feeds into the incubation stage of insight where rich informational chunks are restructured into new understandings. Verbal comprehension and propositional reasoning are examples that demonstrate not all processes associated with intuition and insight are necessarily right hemispheric in origin. Intuition and insight are theoretically well-defined from both a sociocognitive and neural perspective. However, what is required are comprehensive neuropsychological studies directed at determination of the upper performance limits of such functions. Similarly, an understanding of how superior intuition and insight arise over the course of development are nonexistent. As well, further examination of the relationship between intuition and insight to that of other psychometric constructs, such as spatial visualization and general intelligence, are necessary for complete understanding of the area. Finally, improved measures of these constructs in regards to complex social and novel problem solving, are needed.

\section{Conclusions}

The neuropsychological study of right hemisphere language functions has been until very recently been plagued by convoluted misinformation, unquestioned and antiquated assumptions and poorly designed research to examine functions of the right hemisphere in neurologically normal human subjects. As recently as the early 1980's a group of neuropsychologists in Boston and Milan postulated that unique co-localization of cognitive functions within the right hemisphere might possibly lead to the development of anomalous functions. These speculations were not seriously considered until the larger compilations of literature and research were published in the late 1990's including that original contribution by Beeman and Chiarello. ${ }^{2}$ Unfortunately these subsequent approaches studied normal subjects for the most part. Also these studies ignored the fact that with the postulation of anomalous functions was also an 
assumption (perhaps implicit), in the arguments that given base rates of true unambiguous lateralization these subjects would in all probability constitute a highly selective sample. With the exception of publication of a few contemporaneous empirical studies examining these hypotheses, the research field within this area was silent until a decade later. ${ }^{117}$

As the results of this review have shown it is hypothesized that the core anomalous functions that are hypothesized to result from an interaction of high IQ and strong right hemisphere language dominance are exceptional human intuition and insight. Indeed just such cognitive functions or more appropriately "sociocognitive functions" have been postulated to provide a solid empirical and theoretical bridging link between cognitive psychology and social psychological constructs. ${ }^{29}$ Incidentally the discovery of mirror neurons (which has been described as the most important discovery in neuroscience of 1990's), is another bridging link that could ontogenetically speaking augment the development of exceptional intuitive and insightful cognitive functions in atypically localized subjects. ${ }^{274}$ As the review notes the original conceptualization of intuition in Jungian terms may be poorly operationalized and it is now recognized that intuition and insight are currently badly conceived psychometrically and need better operationalizations and standardizations. The exception is that intuition may be closely associated with notions of expertise and Lieberman and colleagues have made excellent progress in elaborating the neurophysiological basis of these terms in cognitive neuroscience parlance. ${ }^{30}$

We note that because these two terms carry with them considerable connotations of "social intelligence" there is good reason to believe that exceptional achievement in scientific, political and/or artistic domains might carry with it such selection effects. Two different types of problem-finding associated with insight are noteworthy in this sense. Presented problems are associated with normal Kuhnian science whereas revolutionary insight is associated with discovered problems of a fundamental paradigm changing, longer-term nature. ${ }^{37}$ Hence well-developed intuition and insight can only be demonstrated in a social context and there are ample casestudies of eminent achievements that show that an abundance of intuition and insight constitute essential ingredients of such achievements. The selection hypothesis also raises the question of the essential ecology of the development of such unique social cognitive functions in humans. The cognitive science literature similarly points towards the importance of unconscious processes in intuition and more declarative conscious processes in insight. This substantial empirical research base alludes to some solid models of intuition and insight as well as theoretical development about how these two "cognitive processes" are interrelated.

For many social psychological, systems neuroscience and cognitive science reasons it is then shown that the core attribute of excellence in intuition consists of efficacy in, and well-developed repertoires of nonverbal decoding behavior. Secondly it is shown that there are also many neurophysiological, behavioral, and gender difference reasons to suggest that women possess superior nonverbal decoding abilities on an average case-by-case basis as compared to most men. Particularly, caudate and orbitofrontal connectivity with the rest of the cortex seem to be able to explain a great deal of data pertaining to basal ganglia neurodegenerative studies and the cognitive neuroscience studies of category learning. It is hypothesized then the caudate may function as a critical waystation between conscious declarative insight-based learning and nonconscious implicit intuitive schemas. Moreover it appears that as a skill becomes routinized the caudate-putamen and its cerebellar connectivity "usurps" control of formerly declarative knowledge schemas enabling: (i) automatization (ii) massively parallel processing and (iii) efficiency in the eventual coordination of multiple informationally-rich schemas. It is then shown how intuition is primary to insight.

We then review how mood and emotion variables may be critically linked to the functioning of neural architectures for intuition and insight. Moreover, intrahemispheric subcortical connectivity within anomalous subjects may figure prominently in the expression of these two sociocognitive phenotypes. In particular, a recently described inferior occipitofrontal fasciculus linking the orbitofrontal, ganglia and posterior cortical regions appears to have the necessary spatiotemporal properties to link intuitive and insight neural architectures. ${ }^{123,124}$ Next the neural correlates of intuition and insight are described. Intuition appears to be dependent on dual hemispheric inferior parietal and caudate structures whereas insight is dependent on right hemispheric temporal and right inferior prefrontal structures. Moreover several key functional neuroimaging studies show the right caudate acts as a way-station directing nonconscious activity along automatic default processing networks unless novel stimuli require frontotemporal evaluation of conscious signals. These signals are simultaneously monitored online for their affective salience in the orbitofrontal cortex. Also this caudate motor skill network is associated with judgments pertaining to "self in high-expertise domains" and thus seems to possess the necessary properties to be associated with ego functions. Finally the use of scripts by subjects in functional MRI and lesion studies illustrates the complex terminal end of the 
ventral stream within the right orbitofrontal cortex. This ventral semantic stream seems to possess all the requisite neuropsychological properties to function as a fast conduit between posterior right inferior parietal nonverbal decoding networks and anterior right orbital highly specialized socialcontext parsing neural networks (eg, script readers).

We then endeavor to show that crossed aphasia (eg, subjects with significant right perisylvian lesions and aphasia implying strong premorbid right hemisphere language dominance), was presumably the only means of finding such rare subjects until the first functional neuroimaging PET studies were published in the late 1980's. It is then shown that recent comprehensive cataloguing of such patients contradicts "the crowding hypothesis". The crowding hypothesis is traditionally held as a truism among many clinical neuropsychologists and it suggests that with unusual atypical dominance often visuospatial functions are co-extensive with language. As a result of this unusual co-localization the crowding hypothesis suggests that performance in both cognitive function domains is substandard to that of traditionally organized left hemisphere language dominant subjects. Also strong neuropsychological data converge on the common conclusion that language is a much more distributed organization in the right hemisphere with perhaps better situational linkages between concepts represented in a much more distantlyrelated manner. Next the relationship between hand praxis and atypical language localization is examined with some interesting attending hypotheses about sex-linked patterns of phenotypic expression.

Based on all the arguments and evidence discussed up until this point, the hypothesis is advanced that the resting state of the anomalous subject is biased towards fundamentally different computations that have been sometimes described as: global, coordinate, imagery, paradigmatic, simultaneous, parallel, holistic, and situational. Several strongly right hemisphere dominant subjects identified through carotid Doppler studies were then compared in comprehensive neuropsychological studies with the understanding that this constitutes a small initial sample. Nonetheless in the context of various converging arguments and using diverse methodologies these tentative case study subjects are provocative. Most subjects showed little evidence of crowding with some evidence of disproportioned development of cognitive abilities. Typically IQ was lower than expected (given achievement indicators), in anomalous subjects and the pattern was reliable enough to suggest a trend. Interestingly most of these subjects were women which is congruent with some of the genetic hypotheses previously advanced. It is then hypothesized (although by no means proven), that the reason that these women seem to be able to achieve beyond conventionally measured IQ is that they possess disproportionately well-developed intuitive and insight based acumen. Finally, a model of the interaction of intuition and insight processes in clinical psychology is advanced with the goal of showing how important these sociocognitive functions are in the real-world.

In the final discussion all of the section's main themes are reviewed integratively with the goal towards to advancing overarching hypotheses as well as testable empirical questions in subsequent studies. The focus is upon determining what these anomalous functions are in the most general sense, as well as why these sociocognitive functions may have evolved, and why they may be so specialized in these rare individuals? We review how the anomalous functions in question seem to have high correlations with both general IQ as well as spatial IQ suggestive of significant intermingling of gene pools. It is shown that high intuition and insight are highly correlated with performance in a host of occupations and that the putative maturation of a right ventral hemispheric "when pathway" is congruent with much recent work in cognitive neuroscience. It is speculated that one of the functions of the development of this neural network would be to facilitate "inverse cognitive modeling" amounting to a neural mechanism for a much more articulate mapping of visual gestures. This would facilitate evolutionarily genetic dispersal as well as enable cross-linguistic fluency and hence facilitate cooperative behaviors across diverse genetic groups. Finally, the unique structure of this well-developed archetypal neural network might also enable highly accurate and precise lexicosemantic mapping of concepts to abstract features of transitive and intransitive human actions through massively parallel integration of information between cortical and subcortical structures.

\section{Limitations}

This lengthy review provides one of the first comprehensive neuropsychological overviews in the literature on "intuition" and "insight" as inter-dependent higher-order cognitive and social constructs. However a recent meta-analytic review of popular notions of "creativity" and hemispheric specialization ${ }^{271}$ may bridge some of the achievement-ability connections not discussed in depth in this article. It has been proposed that intuition and insight are: (1) individual difference variables with a normal distributions (2) are culturally universal constructs (3) are evolutionary advantageous in terms of making social predictions and generating novel solutions to complex social problems (4) have a 
hierarchical structure involving various components that are neuroanatomically localized (5) are predominately lateralized to the right hemisphere (6) are facilitated by co-localization with complimentary functions such as those involved in language processing (7) can account for elevated individual achievement in the context of average intellectual abilities, particularly in individuals with right hemisphere dominance for language. It is important to stress that the results of this review are not meant to naively imply that the left hemisphere and right hemisphere processing styles are absolute in nature. ${ }^{272}$ Instead for some highly selected individuals the left and right hemispheres may possess relatively greater specialization for certain types of spatiotemporal and semantic processes. The two sides of the brain do not operate independently or in isolation from the computations of the other hemisphere. ${ }^{188}$ As with any new foray into the arena of inquiry it is important to stress that this work is meant to facilitate subsequent research and especially further future well-designed empirical studies on the topic.

There is currently insufficient evidence showing how co-localization of cognitive functions within a hemisphere result in either (i) facilitation (as posited in this review) or (ii) interference effects in terms of performance exemplified by the concept of crowding. We have deliberately avoided discussions of crosscultural studies of intuition and insight lest this primarily neuropsychologically-orientated review become unmanageable. There is at present insufficient good measures of intuition and insight and these constructs are at present very poorly psychometrically conceived. We focused on a very small number of studies and in fact the only reason these cases were included was to highlight the usefulness of the systematic neuropsychological study of atypical subjects especially with the rise of advanced structural and functional neuroimaging methods. Also, the cases in question used occupational attainment alone as an indicator of social ascendancy and socioeconomic status and included no measures of social competence, family connections, work ethos, and contemporary motivational constructs. Nor have the base rates of IQ distributions in various occupations been examined. The author has left it to future investigators to discover why if patent right hemisphere dominance is so potentially advantageous why is it also so universally rare? Finally, readers should be aware that even if the hypotheses of a right hemisphere language dominant specialization is not borne out by the data in those subjects with IQ's in the normal range (eg, 100-120) this does not mean that this hypothesis does not apply to subjects above an IQ threshold or of high IQ. This "selection effect" caveat is due to the fact that right hemisphere dominant subjects: (i) constitute a highly selected sample to begin with; (ii) high IQ would allow for more synthetic processes to occur between building blocks of intuition and insight ${ }^{225}$ and (iii) the focus of this review is on abilities posited to allow for exceptional human performance currently at the upper bounds of our understanding of social cognition and human inter-group communication processes.

\section{Acknowledgments}

Simon McCrea, PhD was a Post-Doctoral Fellow and Research Associate of the Divisions of Neurology and Neuroophthalmology within the Faculty of Medicine at the University of British Columbia for part of the duration during which this manuscript was prepared. The author acknowledges the funding and indirect support of a US National Institute of Mental Health (NIMH) R01-MH069898 grant to Jason Barton, $\mathrm{MD}, \mathrm{PhD}$ during formulation of ideas and writing of portions of this review from 2006-2007. Parts of this manuscript were presented at the VII Annual Meeting of the Vision Sciences Society in Sarasota, Florida from May 11 th to May 16th, 2007. The author thanks three anonymous reviewers for their excellent comments. The author reports no conflict of interest in this work.

\section{References}

1. Lindell AK. In your right mind: Right hemisphere contributions to language processing and production. Neuropsychol Rev. 2006;16:131-148.

2. Beeman M, Chiarello C, editors. Right Hemisphere Language Comprehension: Perspectives from Cognitive Neuroscience. Mahwah, NJ: Erlbaum; 1998.

3. Luria AR. Higher Cortical Functions in Man. New York, NY: Basic Books; 1966.

4. Lenneberg E. Biological Foundations of Language. New York, NY: Wiley; 1967.

5. Floel A, Jansen A, Deppe M, et al. Atypical hemispheric dominance for attention: Functional MRI topography. J Cereb Blood F Met. 2005;25:1197-1208.

6. Chee MW, Buckner RL, Savoy RL. Right hemisphere language in a neurologically normal dextral: A fMRI study. Neuroreport. 1998;9:3499-3502.

7. Chee MWL, Caplan D. Face encoding and psychometric testing in healthy dextrals with right hemisphere language. Neurology. 2002;59:1928-1934.

8. Floel A, Knecht S, Lohmann H, et al. Language and spatial attention can lateralize to the same hemisphere in healthy humans. Neurology. 2001;57:1018-1024.

9. Mandonnet E, Nouet A, Gatignol P, Capelle L, Duffau H. Does the left inferior longitudinal fasciculus play a role in language? A brain stimulation study. Brain. 2007;130:623-629.

10. Fiore SM, Schooler JW. Right hemisphere contributions to creative problem solving: Converging evidence for divergent thinking. In: Beeman M, Chiarello C, editors. Right Hemisphere Language Comprehension: Perspectives from Cognitive Neuroscience. Mahwah, NJ: Lawrence Erlbaum; 1998. p. 349-371. 
11. Peterson MA, Rhodes G, editors. Perception of Faces, Objects, and Scenes: Analytic and Holistic Processes. New York, NY: Oxford University Press; 2003.

12. Basso A, Lecours AR, Moraschini S, Vanier M. Anatomoclinical correlations of the aphasias as defined through computerized tomography: Exceptions. Brain Lang. 1985;26:201-229.

13. Fischer RS, Alexander MP, Gabriel C, Gould E, Milione J. Reversed lateralization of cognitive functions in right-handers: Exceptions to classical aphasiology. Brain. 1991;114:245-261.

14. Yin RK. Case Study Research: Design and Methods - Second Edition. London, UK: Sage Publications; 1994.

15. Stemmer B. Neuropragmatics: Disorder and neural systems. In: Stemmer B, Whitaker HA, editors. Handbook of the Neuroscience of Language. San Diego, CA: Academic Press; 2008. p. 175-187.

16. Shallice T. From Neuropsychology to Mental Structure. Cambridge, UK: Cambridge University Press; 1988.

17. Heilman K. Creativity and the Brain. New York, NY: Psychology Press; 2005.

18. Crowne DP. Personality Theory. New York, NY: Oxford University Press; 2007.

19. Jung CG. The relation between the ego and the unconscious. In: Jung CG, Collected Works - Volume 7. Princeton, NJ: Princeton University Press; 1966.

20. Pearsall J. Concise Oxford English Dictionary. New York, NY: Oxford University Press; 2002.

21. Bugental JF, Wegrocki JJ, Murphy G, et al. Symposium on Karl Buhler's contribution to psychology. J Gen Psychol. 1966;75:181-219.

22. Wagner U, Gais S, Haider H, et al. Sleep inspires insight. Nature. 2004;22:352-355.

23. Bowden EM, Jung-Beeman M, Fleck J, Kounios J. New approaches to demystifying insight. Trends Cogn Sci. 2005;9:321-328.

24. Knoblich G, Ohlsson S, Raney GE. An eye movement study of insight problem solving. Mem Cognition. 2001;29:1000-1009.

25. Hogarth RM. Educating Intuition. Chicago, IL: University of Chicago Press; 2001.

26. Volz KG, von Cramon DY. What neuroscience can tell about intuitive processes in the context of perceptual discovery. J Cogn Neurosci. 2006;18:2077-2087.

27. Bechara A, Damasio AR. The somatic marker hypothesis: A neural theory of economic decision. Game Econ Behav. 2005; $52: 336-372$.

28. Myers IB, McCauley MH. Manual: A Guide to Development and Use of the Myers-Briggs Type Indicator. Palo Alto, CA: Consulting Psychologists Press; 1986.

29. Hodgkinson GP, Langan-Fox J, Sadler-Smith E. Intuition: A fundamental bridging construct in the behavioural sciences. Brit J Psychol. 2008;99:1-27.

30. Lieberman MD. Intuition: A social cognitive neuroscience approach. Psychol Bull. 2000;126:109-137.

31. Hodgkinson GP, Clarke I. Exploring the cognitive significance of organizational strategizing: A dual-process framework and research agenda. Hum Relat. 2007;60:243-255.

32. Bruner J. The Process of Education. Cambridge, MA: Harvard University Press; 1960.

33. Chase WG, Simon HA. Perception in chess. Cogn Psychol. 1973; 4:55-81.

34. Ericsson KA, Charness N. Expert performance: Its structure and acquisition. Am Psychol. 1994;49:725-747.

35. Cutica I, Bucciarelli M, Bara BG. Neuropragmatics: Extralinguistic pragmatic ability is better preserved in left-hemisphere-damaged patients than in right- hemisphere-damaged patients. Brain Lang. 2006;98:12-25.

36. Csikszentmihalyi M, Sawyer K. Creative insight: The social dimension of a solitary moment. In: Sternberg RJ, Davidson JE, editors. The Nature of Insight. Cambridge, MA: The MIT Press; 1995. p. 329-363.

37. Kuhn TS. The Structure of Scientific Revolutions. Chicago, IL: University of Chicago Press; 1962.
38. Getzels JW, Csikszentmihalyi M. The Creative Vision. New York, NY: Wiley; 1976.

39. Goel V, Grafman J. Role of the right prefrontal cortex in ill-structured planning. Cogn Neuropsychol. 2000;17:415-436.

40. Wallas G. The Art of Thought. New York, NY: Harcourt Brace Jovanich; 1926.

41. Metcalfe J, Wiebe D. Intuition in insight and noninsight problem solving. Mem Cognition. 1987;15:238-246.

42. Stuss DT, Benson DF. The frontal lobes and language. In: Goldberg E, editor. Contemporary Neuropsychology and the Legacy of Luria. Hillsdale, NJ: Lawrence Erlbaum; 1990:29-49.

43. Dorfman J, Shames VA, Kilhstrom JF. Intuition, incubation, and insight: Implicit cognition in problem solving. In: Underwood G, editor. Implicit Cognition. Oxford, UK: Oxford University Press; 1996. p. 257-296.

44. Merikle PM, Reingold EM. Measuring unconscious perceptual processes. In: Bornstein RF, Pittman TS, editors. Perception Without Awareness. New York, NY: Guilford Press; 1992. p. 55-80.

45. Bowers KS, Farvolden P, Mermigis L. The intuitive antecedents of insight. In: Smith SM, Ward TM, Finske RA, editors. The Creative Cognition Approach. Cambridge, MA: MIT Press; 1995. p. 27-52.

46. Mednick SA, Mednick MT. Examiner's Manual: Remote Associates Test. Boston: Houghton Mifflin; 1967.

47. Topolinski S, Strack F. The architecture of intuition: Fluency and affect determine intuitive judgments of semantic and visual coherence and judgments of grammaticality in artificial grammar learning. $J$ Exp Psychol Gen. 2009;138:39-63.

48. Topolinski S, Strack F. Scanning the "Fringe" of consciousness: What is felt and what is not felt in intuitions about semantic coherence. Conscious Cogn. 2009;18:608-618.

49. Anderson JR. A spreading activation theory of memory. J Verb Learn Verb Be. 1983;22:261-295.

50. Beeman M, Friedman RB, Grafman J, Perez E, Diamond S, Lindsay MB. Summation priming and coarse semantic coding in the right hemisphere. J Cogn Neurosci. 1994;6:26-45.

51. Biederman I. Recognition-by-components: A theory of human image understanding. Psychol Rev. 1987;94:115-147.

52. Tarr MJ. Visual object recognition: Can a single mechanism suffice? In: Peterson MA, Rhodes G, editors. Perception of Faces, Objects, and Scenes: Analytic and Holistic Processes. New York, NY: Oxford University Press; 2003. p. 177-211.

53. Farah MJ. The neural basis of mental imagery. In: Gazzaniga MS, editor. The New Cognitive Neurosciences - Second Edition. Cambridge, MA: MIT Press; 2000:965-974.

54. Fulgosi A, Guilford JP. Short-term incubation in divergent production. Am J Psychol. 1968;81:241-246.

55. Patrick AS. The role of ability in creative "incubation". Pers Indiv Differ. 1986;7:169-174.

56. Ryan JJ, Lopez SJ. Wechsler Adult Intelligence Scale - III. In: Dorfman WI, Hersen M, editors. Understanding Psychological Assessment. New York, NY: Kluwer; 2001. p. 19-42.

57. Barron F, Harrington DM. Creativity, intelligence and personality. Ann Rev Psychol. 1981;32:439-476.

58. Damasio AR. Descartes Error: Emotion, Reason and the Human Brain. New York, NY: Putnam; 1994.

59. Valentine C. The relative reliability of men and women in intuitive judgements of character. Brit J Psychol. 1929;19:213-238.

60. Borod J. Emotion and the brain - Anatomy and theory. An introduction to the special section. Neuropsychology. 1993;7:445-463.

61. Heilman K, Valenstein E, editors. Clinical Neuropsychology - Fourth Edition. New York, NY: Oxford University Press; 2003.

62. Chaminade T, Decety J. Leader or follower? Involvement of the inferior parietal lobule in agency. Neuroreport. 2002;13: 1975-1978.

63. Coslett HB, Saffran EM, Schwoebel J. Knowledge of the human body: a distinct semantic domain. Neurology. 2002;59:357-363. 
64. Farrer C, Franck N, Georgieff N, Frith CD, Decety J, Jeannerod M. Modulating the experience of agency: A positron emission tomography study. Neuroimage. 2003;18:324-333.

65. Leube DT, Knoblich G, Erb M, Grodd W, Bartels M, Kircher TT. The neural correlates of perceiving one's own movements. Neuroimage. 2003;20:2084-2090.

66. Shimada S, Hiraka K, Oda I. The parietal role in the sense of self-ownership with temporal discrepancy between visual and proprioceptive feedbacks. Neuroimage. 2005;24:1225-1232.

67. Decety J, Lamm C. The role of the right temporoparietal junction in social interaction: How low-level computational processes contribute to meta-cognition. Neuroscientist. 2007;13:580-593.

68. Ogawa $\mathrm{K}$, Inui $\mathrm{T}$. Lateralization of the posterior parietal cortex for internal monitoring of self-versus externally generated movements. J Cogn Neurosci. 2007;19:1827-1835.

69. McCrea SM. A functional magnetic resonance imaging study of the body schema using full human line-drawing figures in an on-line verbal naming and localization task of single body part words. Behav Brain Res. 2007; 180:235-240.

70. Corradi-Dell'acqua C, Ueno K, Ogawa A, Cheng K, Rumiati RI, Iriki A Effects of shifting perspective of the self: An fMRI study. Neuroimage. 2008;40:1902-1911.

71. Farrer C, Frey SH, Van Horn JD, et al. The angular gyrus computes action awareness representations. Cereb Cortex. 2008;18:254-261.

72. Ambady N, Rosenthal R. Thin slices of expressive behaviour as predictors of interpersonal consequences: A meta-analysis. Psychol Bull. 1992;111:256-274.

73. DePaulo BM. Nonverbal behaviour and self-presentation. Psychol Bull. 1992;111:203-243.

74. Barr CL, Kleck RE. Self-other perception of the intensity of facial expressions of emotion: Do we know what we show? JPers Soc Psychol. 1995;68:608-618.

75. Schlenker BR, Weigold MF. Interpersonal processes involving impression regulation and management. Ann Rev Psychol. 1992;43:133-168.

76. Reber AS. Implicit learning of artificial grammars. J Verb Learn Verb Be. 1967;6:855-863.

77. Knowlton BJ, Ramus SJ, Squire LR. Intact artificial grammar learning in amnesia: Dissociations of classification learning and explicit memory for specific instances. Psychol Sci. 1992;3:172-179.

78. Dienes Z, Altmann G. Transfer of implicit knowledge across domains: How implicit and how abstract? In: Berry D, editor. How Implicit is Implicit Learning? New York, NY: Oxford University Press; 1997. p. 107-123.

79. Manza L, Reber AS. Representing artificial grammars: Transfer across stimulus forms and modalities. In: Berry D, editor. How Implicit is Implicit Learning? New York, NY: Oxford University Press; 1997. p. 73-106.

80. Martin WR, Stoessl AJ, Adam MJ, et al. Positron emission tomography in Parkinson's disease: Glucose and DOPA metabolism. Adv Neurol. 1987;45:95-98.

81. Alexander GE, DeLong MR, Strick PL. Parallel organization of functionally segregated circuits linking basal ganglia and cortex. Annu Rev Neurosci. 1986;9:357-381.

82. Willingham DB, Koroshetz WJ, Treadwell JR, Bennett JP. Comparisons of Huntington's and Parkinson's disease patients' use of advance information. Neuropsychology. 1995;9:39-46.

83. Berent S, Giordini B, Lehtinen S, et al. Positron emission tomographic scan investigations of Huntington's disease: Cerebral metabolic correlates of cognitive function. Ann Neurol. 1988;23:541-546.

84. Knowlton BJ, Squire LR, Paulsen JS, Swerdlow NR, Swenson M, Butters N. Dissociations within nondeclarative memory in HD. Neuropsychology. 1996;10:538-548.

85. McClelland JL, McNaughton B, O'Reilly RC. Why there are complementary learning systems in the hippocampus and the neocortex: Insights from the successes and failures of connectionist models of learning and memory. Psychol Rev. 1995;102: 419-457.
86. Ashby FG, Alfonso-Reese A, Turken AU, Waldron EM. A neuropsychological theory of multiple systems in category learning. Psychol Rev. 1998;105:442-481.

87. Haggard P, editor. Sensorimotor Foundations of Higher Cognition: Attention and Performance XXII. New York, NY: Oxford University Press; 2007.

88. Bargh JA. Conditional automaticity: Varieties of automatic influence in social perception and cognition. In: Uleman JS, Bargh JA, editors. Unintended Thought. New York, NY: Guilford; 1989. p. 3-51.

89. Bartlett JC, Searcy JH, Abdi H. What are the routes to face recognition? In: Peterson MA, Rhodes G, editors. Perception of Faces, Objects, and Scenes: Analytic and holistic processes. New York, NY: Oxford University Press; 2003. p. 21-52.

90. Jackson GM, Jackson SR, Harrison J, Henderson L, Kennard C. Serial reaction time learning and Parkinson's disease: Evidence for a procedural learning deficit. Neuropsychologia. 1995: 577-593.

91. Knopman D, Nissen MJ. Procedural learning is impaired in HD: Evidence from the serial reaction time task (SRT). Neuropsychologia. 1991;29:245-254.

92. Knopman D, Nissen MJ. Implicit learning in patients with probable Alzheimer's disease. Neurology. 1987;37:784-788.

93. Dominey PF, Ventre-Dominey J, Broussolee E, Jeannerod M. Analogical transfer is effective in a serial reaction time task in Parkinson's disease: Evidence for a dissociable form of sequence learning. Neuropsychologia. 1996;35:1-9.

94. Poldrack RA, Prabhakaran V, Seger CA, Gabrieli JD. Striatal activation during acquisition of a cognitive skill. Neuropsychology. 1999; 13:564-574.

95. Lieberman MD, Chang GY, Chiao J, Bookheimer SY, Knowlton BJ. An event-related fMRI study of artificial grammar learning in a balanced chunk strength design. J Cogn Neurosci. 2004;16: $427-438$.

96. Koriat A, Levy-Sadot R. The combined contributions of the cuefamiliarity and accessibility heuristics to feeling of knowing. $J$ Exp Psychol Learn. 2001;27:34-53.

97. Koriat A. Monitoring one's own knowledge during study: A cueutilization approach to judgments of learning. J Exp Psychol Gen. 1997; 126:349-370

98. Benjamin AS, Bjork RA. Retrieval fluency as a metacognitive index. In: Reder LM, editor. Implicit Memory and Metacognition. Hilldale, NJ: Erlbaum; 1996. p. 309-338.

99. Nelson TO, Narens L. Metamemory: A theoretical framework and new findings. In: Bower G, editor. The Psychology of Learning and Motivation: Advances in Research and Theory. San Diego, CA: Academic Press; 1990. p. 125-173.

100. Betsch T, Plessner H, Schwieren C, Gutug R. I like it but I don't know why: A value-account approach to implicit attitude formation. Pers Soc Psychol B. 2001;27:242-253.

101. Coslett HB. Acquired dyslexia. In: Heilman K, Valenstein E, editors. Clinical Neuropsychology - Fourth Edition. New York, NY: Oxford University Press; 2003. p. 108-125.

102. Snodgrass JG, Vanderwart M. A standardized set of 260 pictures: Norms for name agreement, familiarity, and visual complexity. $J$ Exp Psychol-Hum L. 1980;6:174-215.

103. Wechsler D. Wechsler Memory Scale - Third Edition. San Antonio, TX: Psychological Corporation; 1997.

104. Topolinski S, Strack F. Where there's a will - there's no intuition. The unintentional basis of semantic coherence judgments. J Mem Lang. 2008;58:1032-1048

105. Topolinski S, Strack F. The analysis of intuition: Processing fluency and affect in judgments of semantic coherence. Cognition Emotion. 2009;23:1465-1503.

106. Topolinski S, Likowski KU, Weyers P, Strack F. The face of fluency: Semantic coherence automatically elicits a specific pattern of facial muscle reactions. Cognition Emotion. 2008;23: 260-271. 
107. Schooler JW, Melcher J. The ineffability of insight. In: Smith SM, Ward TB, Fink RA, editors. The Creative Cognition Approach. Cambridge, MA: MIT Press; 1995. p. 97-133.

108. Lazarus RS. Emotion and Adaptation. New York, NY: Oxford University Press; 1991.

109. Morris RG, Robinson RG, Raphael B, Hopwood MJ. Lesion location and poststroke depression. J Neuropsych Clin N. 1996;8:399-403.

110. Bonelli RM, Cummings JL. Frontal-subcortical dementia. Neurologist. 2008;14:100-107.

111. Buchsbaum MS, Wu J, DeLisi LE, et al. Frontal cortex and basal ganglia metabolic rates assessed by positron emission tomography with [18F] 2-deoxyglucose in affective illness. J Affect Disorders. 1986;10:137-152.

112. Cannon DM, Ichise M, Rollis D, et al. Elevated serotonin transporter binding in major depressive disorder assessed using positron emission tomography and [11C] DASB; comparison with bipolar disorder. Biol Psychiat. 2007;62:870-877.

113. Armony JL, LeDoux JE. How danger is encoded: Toward a systems, cellular, and computational understanding of cognitive-emotional interactions in fear. In: Gazzaniga MS, editor. The New Cognitive Neurosciences - Second Edition. Cambridge, MA: The MIT Press; 2000. p. 1067-1079.

114. Hall JA. Nonverbal Sex Differences: Communication Accuracy and Expressive Style. Baltimore, MD: John Hopkins University Press; 1984.

115. Jennings PJ, Janowsky JS. Orwoll E. Estrogen and sequential movement. Behav Neurosci. 1998;112:154-159.

116. Sergent J. Furtive incursion into bicameral minds: Integrative and coordinating role of subcortical structures. Brain. 1990;113:537-568.

117. Clarke JM, Zaidel E. Anatomical-behavioural relationships: Corpus callosum morphometry and hemispheric specialization. Behav Brain Res. 1994;64:185-202.

118. Marzi CA, Perani D, Tassinari G, et al. Pathways of interhemispheric transfer in normals and in a split-brain subject: A positron emission tomography study. Exp Brain Res. 1999;126:451-458.

119. Festinger L. A Theory of Cognitive Dissonance. Evanston, IL: Row Peterson; 1957.

120. Bem DJ. An experimental analysis of self-persuasion. J Exp Soc Psychol. 1965;1:199-218.

121. Lieberman MD, Oscher KN, Gilbert DT, Schacter DL. Do amnesics exhibit cognitive dissonance reduction? The role of explicit memory and attention in attitude change. Psychol Sci. 2001;12:135-140.

122. Ochsner KN, Beer JS, Robertson ER, et al. The neural correlates of direct and reflected self-knowledge. Neuroimage. 2005;28:797-814.

123. Catani M, ffytche DH. The rises and falls of disconnection syndromes. Brain. 2005;128:2224-2239.

124. Bar M, Kassam KS, Ghuman AS, et al. Top-down facilitation of visual recognition. Proc Natl Acad Sci U S A. 2006;103:449-454.

125. Ochsner KN, Ray RD, Cooper JC, et al. For better or for worse: Neural systems supporting the cognitive down- and up-regulation of negative emotion. Neuroimage. 2004;23:483-499.

126. Hariri AR, Bookheimer SY, Mazziotta JC. Modulating emotional responses: Effects of a neocortical network on the limbic system. Neuroreport. 2000;11:43-48.

127. Eslinger PJ, Damasio AR. Severe disturbance of higher cognition after bilateral frontal lobe ablation: Patient EVR. Neurology. 1985;35: 1731-1741.

128. Hare TA, O'Doherty J, Camerer CF, Schultz W, Rangel A. Dissociating the role of the orbitofrontal cortex and the striatum in the computation of goal values and prediction errors. J Neurosci. 2008;28:2630- 5623.

129. Kringelbach ML, Rolls ET. The functional neuroanatomy of the human orbitofrontal cortex: Evidence from neuroimaging and neuropsychology. Prog Neurobiol. 2004;72:341-372.

130. Olson IR, Plotzker A, Ezzyat Y. The enigmatic temporal pole: A review of findings on social and emotional processing. Brain. 2007;130:1718-1731.

131. Crick FC, Koch C. What is the function of the claustrum? Philos T Roy Soc B. 2005;360:1271-1279.
132. Battelli L, Pascual-Leone A, Cavanaugh P. The 'when' pathway of the right parietal lobe. Trends Cogn Sci. 2007;11:204-210.

133. Ilg R, Vogeley K, Goschke T, et al. Neural processes underlying intuitive coherence judgments as revealed by fMRI on a semantic judgment task. Neuroimage. 2007;38:228-238.

134. Bowden EM, Jung-Beeman M. Methods for investigating the neural components of insight. Methods. 2007;42:87-99.

135. Jung-Beeman M, Bowden EM, Haberman J, et al. Neural activity when people solve verbal problems with insight. PLoS Biol. 2004;2:E97.

136. Bowden EM, Beeman MJ. Getting the right idea: Semantic activation in the right hemisphere may help solve insight problems. Psychol Sci. 1998;6:435-440.

137. Sandkuhler S, Bhattacharya J. Deconstructing insight: EEG correlates of insightful problem solving. PLoS One. 2008;3:e1459.

138. Ash IK, Wiley J. The nature of restructuring in insight: An individual differences approach. Psychon B Rev. 2006;13:66-73.

139. Behrmann M, Geng JJ, Shomstein S. Parietal cortex and attention. Curr Opin Neurobiol. 2004;14:212-217.

140. Miller LA, Tippett LJ. Effects of focal brain lesions on visual problem solving. Neuropsychologia. 1996;34:387-398.

141. Goel V, Vartanian O. Dissociating the roles of right ventral lateral and dorsal lateral prefrontal cortex in generation and maintenance of hypothesis in set-shift problems. Cereb Cortex. 2005;15:1170-1177.

142. Bowden EM, Jung-Beeman M. Aha! Insight experience correlates with solution activation in the right hemisphere. Psychon B Rev. 2003;10:730-737.

143. Mazoyer BM, Tzourio N, Frak V, et al. The cortical representation of speech. J Cogn Neurosci. 1993;5:467-479.

144. Stowe LA, Paans AMJ, Wijers AA, et al. Sentence comprehension and word repetition: A positron emission tomography investigation. Psychophysiology. 1999;36:786-801.

145. Seger CA, Desmond JE, Glover GH, et al. FMRI evidence for right hemisphere involvement in processing unusual semantic relationships. Neuropsychology. 2000;14:361-369.

146. Robertson DA, Gernsbacher MA, Guidotti SJ, et al. Functional neuroanatomy of the cognitive process of mapping during discourse comprehension. Psychol Sci. 2000;11:255-260.

147. Frith $\mathrm{CD}$. The role of the dorsolateral prefrontal cortex in the selection of action as revealed by functional imaging. In: Monsell S, Driver J, editors. Control of Cognitive Processes: Attention and Performance XVIII. Cambridge, MA: MIT Press; 2000. p. 549-565.

148. Knoblich G, Ohlsson S, Haider H, Rhenius D. Constraint relaxation and chunk decomposition in insight problem solving. J Exp Psychol Learn. 1999;25:1534-1555.

149. Reverberi C, Toraldo A, D'Agostini S, Skrap M. Better without (lateral) frontal cortex? Insight problem solved by frontal patients. Brain. 2005;128:2882-2890.

150. Feinberg TE, Keenan JP. Where in the brain is the self? Conscious Cogn. 2005;14:661-679.

151. Lieberman MD, Gaunt R, Gilbert DT, Trope Y. Reflection and reflexion: A social cognitive neuroscience approach to attributional inference. Adv Exp Soc Psychol. 2002;34:199-249.

152. Wagner AD, Schacter DL, Rotte M, et al. Building memories: Remembering and forgetting of verbal experiences as predicted by brain activity. Science. 1998;281:1188-1191.

153. Lieberman MD, Pfeifer J. The self and social perception: Three kinds of questions in social cognitive neuroscience. In: Easton A, Emery N, editors. Cognitive Neuroscience of Emotional and Social Behaviour. Philadelphia, PA: Psychology Press; 2005. p. 195-236.

154. Lieberman MD, Jarcho JM, Satpute AB. Evidence-based and intuition-based self-knowledge: An fMRI study. J Pers Soc Psychol. 2004;87:421-435.

155. Klein SB, Loftus J. The mental representation of trait and autobiographical knowledge about the self. In: Srull TK, Wyer Jr RS, editors. The Mental Representation of Trait and Autobiographical Knowledge About the Self. Hillsdale, NJ: Erlbaum; 1993. p. 1-49. 
156. Klein SB, Rozendal K, Cosmides L. A social-cognitive neuroscience analysis of the self. Soc Cognition. 2002;20:105-135.

157. Lieberman MD. Reflective and reflexive judgment processes: A social cognitive neuroscience approach. In: Forgas JP, Williams KR, von Hippel W, editors. Social Judgments: Implicit and Explicit Processes. New York, NY: Cambridge University Press; 2003. p. 44-67.

158. Knowlton BJ, Squire LR. Artificial grammar depends on implicit acquisition of both abstract and exemplar-specific information. $J$ Exp Psychol Learn. 1996;22:169-181.

159. Milne E, Grafman J. Ventromedial prefrontal cortex lesions in humans eliminate implicit gender stereotyping. J Neurosci. 2001;21: RC151-RC156.

160. Garrard P, Hodges JR. Semantic dementia: Clinical, radiological and pathological perspectives. J Neurol. 2000;247:409-422.

161. Kahneman D. A perspective on judgment and choice: Mapping bounded rationality. Am Psychol. 2003;58:697-720.

162. Pretz JE. Intuition versus analysis: Strategy and experience in complex everyday problem solving. Mem Cognit. 2008;36:554-556.

163. Masicampo EJ, Baumeister RF. Toward a physiology of dual-process reasoning and judgment. Psychol Sci. 2008;19:255-260.

164. Van Hesselt VB, Hersen M. Handbook of Social Development: A Lifespan Perspective. New York, NY: Plenum Press; 1992.

165. Grafman J. Experimental assessment of adult frontal lobe function. In: Miller BL, Cummings JL, editors. The Human Frontal Lobes: Functions and Disorder. New York, NY: Guilford Press; 1999. p. 321-344.

166. Sirigu I, Zalla T, Pillon B, Grafman J, Agid Y, Dubois B. Encoding of sequence and boundaries of scripts following prefrontal lesions. Cortex. 1996;32:297-310.

167. Chevingnard M, Pillon B, Pradat-Diehl P, et al. An ecological approach to planning dysfunction: Script execution. Cortex. 2000;36:649-669.

168. Knutson KM, Wood JN, Grafman J. Brain activation in processing temporal sequences: An fMRI study. Neuroimage. 2004;23:1299-1307.

169. Wood JN, Tierney M, Bidwell LA, Grafman J. Neural correlates of script event knowledge: A neuropsychological study following prefrontal injury. Cortex. 2005;41:796-804.

170. Allain P, Verny C, Aubin G, Bonneau D, Dubas F, Le Gall D. Processing of 'scripts' and the frontal lobe function in Huntington's disease. Revue Neurol-France. 2004;60:434-440.

171. Godbout L, Doyon J. Defective representation of knowledge in Parkinson's disease: Evidence from a script-production task. Brain Cognition. 2000;44:490-510.

172. Duffau $\mathrm{H}$. The anatomo-functional connectivity of language revisited: New insights provided by electrostimulation and tractography Neuropsychologia. 2008;46:927-934.

173. Schwartz MF, Buxbaum LJ, Montgomery MW, et al. Naturalistic action production following right hemisphere stroke. Neuropsychologia. 1999;37:51-66.

174. Marien P, Paghera B, De Deyn PP, Vignolo LA. Adult crossed aphasia in dextrals revisited. Cortex. 40:41-74.

175. Jansen A, Floel A, Menke R, Kanowski M, Knecht S. Dominance for language and spatial processing: Limited capacity of a single hemisphere. Neuroreport. 2005;16:1017-1021.

176. Braun CM, Montour-Proulx I, Daigneault S, et al. Prevalence, and intellectual outcome of unilateral focal cortical brain damage as a function of age, sex and aetiology. Behav Neurol. 2001;13:105-116.

177. Knecht S, Drager B, Deppe M, et al. Handedness and hemispheric language dominance in healthy humans. Brain. 2000;123: 2512-2518.

178. Springer JA, Binder JR, Hammeke TA, et al. Language dominance in neurologically normal and epilepsy subjects: A functional MRI study. Brain. 1999;122:2033-2046.

179. Zangwill OL. Two cases of crossed aphasia in dextrals. Neuropsychologia. 1979;17:167-172.

180. Meador KJ, Loring DW, Lee K, et al. Cerebral lateralization: Relationship of language and ideomotor praxis. Neurology. 1999;53:2028-2031.
181. Butcher LM, Kennedy JKJ, Plomin R. Generalist genes and cognitive neuroscience. Curr Opin Neurobiol. 2006;16:145-151.

182. Joanette Y. Aphasia in left-handers and crossed aphasia. In: Boller F, Grafman J, editors. Handbook of Neuropsychology. Amsterdam, The Netherlands: Elsevier; 1989. p. 173-183.

183. Alexander MP, Annett M. Crossed aphasia and related anomalies of cerebral organization: Case reports and a genetic hypothesis. Brain Lang. 1996;55:213-239.

184. Hecaen H, Sauguet J. Cerebral dominance in left-handed subjects. Cortex. 1971;7:19-48.

185. McCarthy RA, Warrington EK. Cognitive Neuropsychology. San Diego, CA: Academic Press; 1990:5-8.

186. Castro-Caldas A, Confraria A, Poppe P. Nonverbal disturbances in crossed aphasia. Aphasiology. 1987;1:403-413.

187. Annett M. Left-handedness as a function of sex, maternal versus paternal inheritance, and report bias. Behav Genet. 1999;29:103-114.

188. Ivry RB, Robertson LC. The Two Sides of Perception. Cambridge, MA: MIT Press; 1998.

189. Schooler JW, Fallshore M, Fiore SM. Putting insight into perspective. In: Sternberg RJ, Davidson JE, editors. The Nature of Insight. Cambridge, MA: MIT Press; 1995. p. 559-587.

190. Schooler JW, Ohlsson S, Brooks K. Thoughts beyond words: When language overshadows insight. J Exp Psychol Gen. 1993;122:166-183.

191. Drewe EA. The effect of type and area of brain lesion on Wisconsin Card Sorting Test performance. Cortex. 1974;10:159-170.

192. Rauch R. Cognitive strategies in patients with unilateral temporal lobe excisions. Neuropsychologia. 1977;15:385-396.

193. Sandson J, Albert ML. Perseveration in behavioural neurology. Neurology. 1987;37:1736-1741.

194. Hermann BP, Wyler AR, Richey ET. Wisconsin Card Sorting Test performance in patients with complex partial seizures of temporal lobe origin. J Clin Neuropsychol. 1988;10:467-476.

195. Ohlsson S. Information-processing explanations of insight and related phenomena. In: Keane M, Gilhooley K, editors. Advances in the Psychology of Thinking. London, UK: Harvester-Wheatsheaf; 1992. p. 1-44

196. Bowers KS, Regehr G, Balthazard C, Parker K. Intuition in the context of discovery. Cogn Psychol. 1990;22:72-110.

197. Dobbins IG, Davachi L. Functional neuroimaging of episodic memory. In: Cabeza R, Kingstone A, editors. Handbook of Functional Neuroimaging of Cognition - Second Edition. Cambridge, MA: The MIT Press; 2006. p. 229-268.

198. Drews E. Qualitatively different organizational structures of lexical knowledge in the left and right hemispheres. Neuropsychologia. 1987;25:419-427.

199. Chiarello C, Burgess C, Richards L, Pollock A. Semantic and associative priming in the cerebral hemispheres: Some words do, some words don't...sometimes, some places. Brain Lang. 1990;38:75-104.

200. Hutlser JJ. The specialized structure of human language cortex: Pyramidal cell size asymmetries within auditory and language associated regions of the temporal lobes. Brain Lang. 2003;86:226-242.

201. Hutsler J, Galuske RAW. Hemispheric asymmetries in cerebral cortical networks. Trends Neurosci. 2003;26:429-435.

202. Behrmann M. Neuropsychological approaches to perceptual organization: Evidence from visual agnosia. In: Peterson MA, Rhodes G, editors. Perception of Faces, Objects, and Scenes: Analytic and Holistic Processes. New York, NY: Oxford University Press; 2003. p. 295-334.

203. Witkin HA, Oltham PK, Raskin E, Karp SA. A Manual for the Embedded Figures Test. Palo Alto, CA: Consulting Psychologists Press; 1971.

204. Paivio A, Yuille JC, Madigan SA. Concreteness, imagery, and meaningfulness values for 925 nouns. $J$ Exp Psychol. 1968;76:1-25.

205. Jakobson R. Studies on Child Language and Aphasia. The Hague, Belgium: Mouton; 1971.

206. Das JP, Kirby JR, Jarman RF. Simultaneous and successive syntheses: An alternative model for cognitive abilities. Psychol Bull. $1975 ; 82: 87-103$. 
207. Navon D. Forest before tress: The precedence of global features in visual perception. Cogn Psychol. 1977;9:353-383.

208. Kosslyn SM, Chabris CF, Marsolek CJ, Koenig O. Categorical and coordinate spatial relations. Computational analyses and computer simulations. J Exp Psychol Human. 1992;18:562-577.

209. Treisman A. Feature binding, attention and object perception. Philos T Roy Soc B. 1998;353:1295-1306.

210. McCrea SM. A review and empirical study of the composite scales of the Das-Naglieri Cognitive Assessment System. Psychol Res Behav Manag. 2009;2:59-79.

211. Baynes K, Gazzaniga MS. Consciousness, introspection, and the splitbrain: The two minds/One body problem. In: Gazzaniga MS, editor. The New Cognitive Neurosciences - Second Edition. Cambridge, MA: MIT Press; 2000. p. 1355-1363.

212. Subramaniam K, Kounios J, Parrish TB, Jung-Beeman M. A brain mechanism for facilitation of insight by positive affect. $J \operatorname{Cogn}$ Neurosci. 2009;21:415-432.

213. Gasper K, Clore GL. Attending to the big picture: Mood and global versus local processing of visual information. Psychol Sci. 2002;13:34-40.

214. Rowe G, Hirsch JB, Anderson AK. Positive affect increases the breadth of attentional selection. Proc Natl Acad Sci U S A. 2007;101:383-388.

215. Cai Q, Lavidor M, Brysbaert M, Paulignan Y, Nazir TA. Cerebral lateralization of frontal lobe language processes and lateralization of the posterior visual word processing systems. J Cogn Neurosci. 2008;20:672-681.

216. Carter CS, McDonald AM, Botvinick M, et al. Parsing executive processes: Strategic vs evaluative functions of the anterior cingulate cortex. Proc Natl Acad Sci U S A. 2000;97:1944-1948.

217. Chambers CD, Payne JM, Stokes MG, Mattingley JB. Fast and slow parietal pathways mediate spatial attention. Nat Neurosci. 2004;7:217-218.

218. Kounios J, Frymiare JL, Bowden EM, et al. The prepared mind: Neural activity prior to problem presentation predicts subsequent solution by sudden insight. Psychol Sci. 2006;17:882-890.

219. Kounios J, Fleck JI, Green DL, et al. The origins of insight in resting-state brain activity. Neuropsychologia. 2008;46: 281-291.

220. Christoff K, Ream JM, Gabrieli JDE. Neural basis of spontaneous thought processes. Cortex. 2004;40:623-630.

221. Gevins A, Smith ME. Neurophysiological measures of working memory and individual differences in cognitive ability and cognitive style. Cereb Cortex. 2000;10:829-839.

222. Carson SH, Peterson JB, Higgins DM. Decreased latent inhibition is associated with increased creative achievement in high-functioning individuals. J Pers Soc Psychol. 2003;85:499-506.

223. St. George M, Kutas M, Martinez A, et al. Semantic integration in reading: Engagement of the right hemisphere during discourse processing. Brain. 1999;122:1317-1325.

224. Kircher TTJ, Brammer M, Andreu NT, Williams SCR, McGuire PK. Engagement of right temporal cortex during linguistic context. Neuropsychologia. 2001;39:798-809.

225. Carroll JB. Human Cognitive Abilities: A Survey of Factor-Analytic Studies. Cambridge, MA: Cambridge University Press; 1993.

226. Knecht S, Deppe M, Ebner A, et al. Determination of hemispheric language dominance: Direct comparison of functional transcranial Doppler sonography with the WADA test. Neuroimage. 1997; 5(4 Pt 2):S586.

227. Knecht S, Drager B, Floel A, et al. Behavioural relevance of atypical language lateralization in healthy subjects. Brain. 2001; 124:1657-1665.

228. Knecht S, Jansen A, Frank A, et al. How atypical is atypical language dominance? Neuroimage. 2003;18:917-927.

229. Posner MI, Walker JA, Friedrich FJ, Rafal RD. Effects of parietal injury on covert orienting of attention. $J$ Neurosci. 1984;4: $1863-1877$.
230. Raven JC. Standard Progressive Matrices Sets A,B,C,D, and E. London, UK: H.K. Lewis; 1958.

231. Nakagawa A. Attentional balance and intelligence. Intelligence. 1996;22:277-290.

232. Kraft RH. The effect of sex, laterality, and familial handedness on intellectual abilities. Neuropsychologia. 1983;21:79-89.

233. Halpern DF, Haviland MG, Killian CD. Handedness and sex differences in intelligence: Evidence from the Medical College Admission Test. Brain Cognition. 1998;38:87-101.

234. Lee KH, Choi YY, Gray JR, et al. Neural correlates of superior intelligence: Stronger recruitment of posterior parietal cortex. Neuroimage. 2006;29:578-586.

235. Warrington EK, James M, Maciejewski C. The WAIS as a lateralizing and localizing diagnostic instrument: A study of 656 patients with unilateral cerebral lesions. Neuropsychologia. 1986; 24:223-239.

236. Deppe M, Knecht S, Papke K, et al. Assessment of hemispheric language lateralization: A comparison between fMRI and fTCD. J Cereb Blood F Met. 2000;20:263-268.

237. Teuber HL. Why two brains? In: Schmitt FO, Worden FG, editors. The Neurosciences - Third Study Program. Cambridge, MA: MIT Press; 1974. p. 71-74.

238. Lezak MD. Neuropsychological Assessment - Third Edition. New York, NY: Oxford University Press; 1995.

239. Marshall RS, Lazar RM, Van Heertum RL, Esser PD, Perera GM, Mohr JP. Changes in regional cerebral blood flow related to line bisection discrimination and visual attention using HMPAO-SPECT. Neuroimage. 1997;6:139-144.

240. Dronkers NF, Knight RT. Right-sided neglect in a left-hander: Evidence for reversed hemispheric specialization of attention capacity. Neuropsychologia. 1989;27:729-7355.

241. Sturm W, Willmes K, Horn W. Leistungsprufsystem fur 18-50 jahrige (LPS). Gottinggen: Hogrefe, Verlag fur Psychologie; 1993.

242. Kaufman AS. Assessing Adolescent and Adult Intelligence. Boston, MA: Allyn and Bacon; 1990.

243. Woods RP, Dodrill CB, Ojemann GA. Brain injury, handedness, and speech lateralization in a series of amobarbital studies. Ann Neurol. 1988;23:510-518.

244. Hiscock M. Brain lateralization across the life span. In: Stemmer B, Whitaker HA, editors. Handbook of Neurolinguistics. San Diego, CA: Academic Press; 1998. p. 357-368.

245. Ogdon DP. Handbook of Psychological Signs, Symptoms, and Syndromes. Los Angeles, CA: Western Psychological Services; 2001.

246. Ogdon DP. Psychodiagnostics and Personality Assessment. Los Angeles, CA: Western Psychological Services; 2004.

247. Hammer EF, editor. Advances in Projective Drawing Interpretation. Springfield, IL: Charles C. Thomas Publishers, Ltd; 1997.

248. Ansburg PI. Individual differences in problem solving via insight. Curr Psychol. 2000;19:143-146.

249. Chu Y. Human Insight Problem Solving: Performance, Processing, and Phenomenology. Unpublished doctoral dissertation. Hawaii, HA: University of Hawaii at Manoa Press; 2008.

250. Fleck JI. The Role of Working Memory in Solving Analytic versus Insight Problems. Unpublished doctoral dissertation. Philadelphia, PA: Temple University Press; 2005.

251. Klein G. Intuition at Work. New York, NY: Bantam Dell; 2003.

252. Hall KH. Reviewing intuitive decision-making and uncertainty: The implications for medical education. Med Educ. 2002;36:216-224.

253. Rehm JT, Gadenne V. Intuitive Predictions and Professional Forecasts: Cognitive Processes and Social Consequences. Oxford, UK: Pergamon; 1990.

254. Benner P, Tanner C. Clinical judgement: How expert nurses use intuition. Am J Nurs. 1987;87:23-31.

255. Sadler E, Shefy E. The intuitive executive: Understanding and applying 'gut feel' in decision making. Acad Manag Exec. 2004; 18:76-91. 
256. Jansen A, Liuzzi G, Deppe M, et al. Structural correlates of functional language dominance: A voxel-based morphometry study. $J$ Neuroimaging. 2009; In Press.

257. Saxe R, Wexler A. Making sense of another mind: The role of the right temporo-parietal junction. Neuropsychologia. 2005;43:1391-1399.

258. Saxe R. Brain regions for theory of mind. Lecture presented at the Summer Institute on Social Cognition. University of Quebec at Montreal, Montreal, Canada; July 2008

259. Samson D, Apperley IA, Kathirgamanathan U, Humphreys GW. Seeing it my way: A case of selective deficit in inhibiting self-perspective. Brain. 2005;128:1102-1111.

260. Uddin LQ, Kaplan JT, Molnar-Szakacs I, et al. Self-face recognition activates a frontoparietal "mirror" network in the right hemisphere: An event-related fMRI study. Neuroimage. 2005;15:926-935.

261. Bottini G, Corcoran R, Sterzi R, et al. The role of the right hemisphere in the interpretation of figurative aspects of language: A positron emission tomography activation study. Brain. 1994;117:1241-1253.

262. Colom R, Jung RE, Haier RJ. General intelligence and memory span: Evidence for a common neuroanatomic framework. Cogn Neuropsychol. 2007;24:867-878.

263. Davidson PR, Wolpert DM. Widespread access to predictive models in the motor system: A short review. J Neural Eng. 2005;2: S313-S319.

264. Ashby FG, Ell SW. The neurobiology of human category learning. Trends Cogn Sci. 2001;5:204-210.

265. McGeough PD, Brang D, Ramachandran VS. Apraxia, metaphor and mirror neurons. Med Hypotheses. 2007;69:1165-1168.
266. Ramachandran VS, Hubbard EM. Synaesthesia: A window into perception, thought and language. J Consciousness Stud. 2001;8:3-34.

267. McCrea SM. Bipolar disorder and neurophysiologic mechanisms. Neuropsychiatr Dis Treat. 2008;4:1129-1153.

268. Allman JM, Watson KK, Tetreault NA, Hakeem AY. Intuition and autism: A possible role for Von Economo neurons. Trends Cogn Sci. 2005;9:367-373.

269. Carlson SM, Moses LJ. Individual differences in inhibitory control and children's theory of mind. Child Dev. 2001;72:1032-1053.

270. Dawson G, Fischer KW, editors. Human Behaviour and the Developing Brain. New York, NY: The Guilford Press; 1994.

271. Mihov KM, Denzler M. Hemispheric specialization: Creativity revisited. A meta-analytic review of lateralization of creativity. In Press.

272. KolbB, Whishaw IQ.Fundamentals ofHuman Neuropsychology-Fourth Edition. New York, NY: W.H. Freeman and Company; 1996.

273. Gluck MA, Shohamy D, Myers C. How do people solve the 'weather prediction' task?: Individual variability in strategies for probabilistic category learning. Learn Memory. 2002;9:408-418.

274. Westbury CF, McCrea SM, Binder JR. Is there privileged access to semantics for mirror neuron related verbs: A functional magnetic resonance imaging study. Brain Cognition. 2006;60:322.

275. Wildman FW, Wildman RWII, Smith RD. Expansiveness-constriction on the H-T-P as indicators of extraversion-introversion. J Clin Psychol. $1963 ; 23: 493-494$

\section{Publish your work in this journal}

Psychology Research and Behavior Management is an international, peerreviewed, open access journal focusing on the science of psychology and its application in behavior management to develop improved outcomes in the clinical, educational, sports and business arenas. Specific topics covered include: Neuroscience, memory \& decision making; Behavior

\section{Dovepress}

modification \& management; Clinical applications; Business \& sports performance management; Social and developmental studies; Animal studies. The manuscript management system is completely online and includes a quick and fair peer-review system. Visit http://www.dovepress. com/testimonials.php to read real quotes from published authors. 